\title{
New ATCA, ALMA and VISIR observations of the candidate LBV SK -67 266 (S61): the nebular mass from modelling 3D density distributions
}

\author{
C. Agliozzo, ${ }^{1,2 \star}$ R. Nikutta, ${ }^{3,4 \star}$ G. Pignata, ${ }^{2,1}$ N. M. Phillips, ${ }^{5,6}$ A. Ingallinera, ${ }^{7}$ \\ C. Buemi, ${ }^{7}$ G. Umana,${ }^{7}$ P. Leto,${ }^{7}$ C. Trigilio, ${ }^{7}$ A. Noriega-Crespo,${ }^{8}$ R. Paladini, ${ }^{9}$ \\ F. Bufano ${ }^{7}$ and F. Cavallaro ${ }^{7,10,11}$ \\ ${ }^{1}$ Millennium Institute of Astrophysics (MAS), Nuncio Monseñor Sótero Sanz 100, Providencia, Santiago, Chile \\ ${ }^{2}$ Departamento de Ciencias Fisicas, Universidad Andres Bello, Avda. Republica 252, Santiago, 8320000, Chile \\ ${ }^{3}$ National Optical Astronomy Observatory, 950 N Cherry Ave, Tucson, AZ 85719, USA \\ ${ }^{4}$ Instituto de Astrofísica, Facultad de Física, Pontificia Universidad Católica de Chile, Casilla 306, Santiago 22, Chile \\ ${ }^{5}$ European Southern Observatory, Alonso de Córdova 3107, Vitacura, Santiago, Chile \\ ${ }^{6}$ Joint ALMA Observatory, Alonso de Córdova 3107, Vitacura, Santiago, Chile \\ ${ }^{7}$ INAF-Osservatorio Astrofisico di Catania, Via S. Sofia 78, I-95123 Catania, Italy \\ ${ }^{8}$ Space Telescope Science Institute 3700 San Martin Dr., Baltimore, MD, 21218, USA \\ ${ }^{9}$ Infrared Processing Analysis Center, California Institute of Technology, 770 South Wilson Ave., Pasadena, CA 91125, USA \\ ${ }^{10}$ CSIRO Astronomy and Space Science, PO Box 76, Epping, NSW 1710, Australia \\ ${ }^{11}$ International Centre for Radio Astronomy Research, Curtin University, Bentley, WA 6102, Australia
}

Accepted 2016 November 15. Received 2016 November 14; in original form 2016 October 13

\begin{abstract}
We present new observations of the nebula around the Magellanic candidate Luminous Blue Variable S61. These comprise high-resolution data acquired with the Australia Telescope Compact Array (ATCA), the Atacama Large Millimetre/Submillimetre Array (ALMA), and the VLT Imager and Spectrometer for mid Infrared (VISIR) at the Very Large Telescope. The nebula was detected only in the radio, up to $17 \mathrm{GHz}$. The $17 \mathrm{GHz}$ ATCA map, with 0.8 arcsec resolution, allowed a morphological comparison with the $\mathrm{H} \alpha$ Hubble Space Telescope image. The radio nebula resembles a spherical shell, as in the optical. The spectral index map indicates that the radio emission is due to free-free transitions in the ionized, optically thin gas, but there are hints of inhomogeneities. We present our new public code RHOcube to model 3D density distributions and determine via Bayesian inference the nebula's geometric parameters. We applied the code to model the electron density distribution in the S61 nebula. We found that different distributions fit the data, but all of them converge to the same ionized mass, $\sim 0.1 \mathrm{M}_{\odot}$, which is an order of magnitude smaller than previous estimates. We show how the nebula models can be used to derive the mass-loss history with high-temporal resolution. The nebula was probably formed through stellar winds, rather than eruptions. From the ALMA and VISIR non-detections, plus the derived extinction map, we deduce that the infrared emission observed by space telescopes must arise from extended, diffuse dust within the ionized region.
\end{abstract}

Key words: methods: statistical-circumstellar matter-stars: individual: (SK-67 266)stars: massive-stars: mass-loss - radio continuum: stars.

\section{INTRODUCTION}

Luminous Blue Variables (LBVs) are evolved massive stars $\left(>20 \mathrm{M}_{\odot}\right)$, intrinsically bright $\left(L \sim 10^{5}-10^{6.3} \mathrm{~L}_{\odot}\right)$ and hot $(\mathrm{O}$, $\mathrm{B}$ spectral type). They are unstable and exhibit spectroscopic and photometric variability. During the LBV variability cycle, they can resemble a cooler supergiant of spectral type A or F and show visual magnitude variations over a wide range of amplitudes and timescales (as discussed and reviewed by Humphreys \& Davidson 1994; van Genderen 2001). Because of their instability, they suffer massloss at high rate $\left(\dot{M} \gtrsim 10^{-5} \mathrm{M}_{\odot} \mathrm{yr}^{-1}\right)$ and form circumstellar nebulae. The mechanism that causes this instability is still poorly understood. To explain the common 'S Doradus type' outbursts 
(with visual magnitude variations of 1-2 mag on time-scales of years), changes of the photospheric physical conditions have been invoked. This variation of the photospheric physical conditions is caused by a change of the wind efficiency due to variation of the ionization of $\mathrm{Fe}$, which is the main carrier of line-driven stellar winds. This mechanism is known as the 'bi-stability jump' (explained by Pauldrach \& Puls 1990; Lamers, Snow \& Lindholm 1995), a predicted effect of which is mass-loss variability (Vink, de Koter \& Lamers 1999). The observational mass-loss rates estimated from different indicators (e.g. UV and optical emission lines, radio free-free emission) have often been discrepant, most of the time depending on whether clumped or unclumped wind models were assumed. For example, Fullerton, Massa \& Prinja (2006) found that mass-loss rates estimated from $\mathrm{P} v$ lines in clumpy stellar winds of $\mathrm{O}$ stars are systematically smaller than those obtained from squared electron density diagnostics (e.g. $\mathrm{H}_{\alpha}$ and radio free-free emission) with unclumped wind models, resulting in empirical mass-loss rates overestimated by a factor of 10 or more. The implication is that line-driven stellar winds are not sufficient to strip off quickly the $\mathrm{H}$ envelope, before they evolve to Wolf-Rayet (WR) stars (Conti \& Frost 1976). Enhanced mass-loss was therefore proposed to reduce the stellar mass, possibly through short-duration eruptions or explosions (Humphreys \& Davidson 1994; Smith \& Owocki 2006). Subsequently, Oskinova, Hamann \& Feldmeier (2007) showed that if macro-clumping (instead of optically thin, micro-clumping) is taken into account, $\mathrm{P} v$ lines become significantly weaker and lead to underestimation of the mass-loss rate. Finally, Vink \& Gräfener (2012) showed that for moderate clumping (factor up to 10) and reasonable mass-loss rate reductions (of a factor of 3 ), the empirical mass-loss rates agree with the observational rates and, more importantly, with the model-independent transition mass-loss rate that is independent of any clumping effects. The implication of this is that eruptive events are not needed to make WR stars.

The mechanism that triggered the 'giant eruptions' (with visual magnitude changes larger than 2 mag) witnessed in the 17th (P Cygni) and in the 19th century ( $\eta$ Carinae) in our Galaxy is still unknown, but some scenarios involving hydrodynamic (sub-photospheric) instabilities, rapid rotation, and close binarity have been proposed (e.g. Humphreys \& Davidson 1994, and ref. therein). The presence of nebulae in most of the known objects (e.g. Humphreys \& Davidson 1994; van Genderen 2001; Clark, Larionov \& Arkharov 2005) suggests that these are a common aspect of the LBV behaviour (Weis 2008).

Given the short duration of the LBV phase $\left(10^{4}-10^{5} \mathrm{yr}\right)$, combined with the rapid evolution of massive stars, LBVs are rare: only a few tens of objects in our Galaxy and in the Magellanic Clouds (Davidson \& Humphreys 2012) satisfy the variability criteria coupled with high mass-loss rates (Humphreys \& Davidson 1994). Nevertheless, based on the discovery of dusty ring nebulae surrounding luminous stars, the number of Galactic candidate LBVs (cLBVs) has increased recently to 55 (Gvaramadze, Kniazev \& Fabrika 2010; Wachter et al. 2011; Nazé, Rauw \& Hutsemékers 2012). A few tens of confirmed LBVs have been discovered in farther galaxies (e.g. M31, M33, NGC2403; Humphreys et al. 2016, and ref. therein).

LBV ejecta are the fingerprints of the mass-loss phenomenon suffered by the star. The LBV nebulae (LBVNe) observed in our Galaxy usually consist of both gas and dust. Previous studies of known Galactic LBVs at radio wavelengths, which trace the ionized component, estimated the masses of the nebulae and their current mass-loss rates (e.g. Duncan \& White 2002; Lang et al. 2005; Umana et al. 2005, 2010, 2011a, 2012; Buemi et al. 2010; Agliozzo et al. 2012, 2014; Paron et al. 2012; Buemi et al. 2017). On the other hand, infrared (IR) observations revealed that the dust is often distributed outside of the ionized region, indicative of mass-loss episodes of different epochs and/or that the nebulae are ionizationbounded (e.g. G79.29+0.46, G26.47+0.02, Wray 15-751, AG Car; Kraemer et al. 2010; Jiménez-Esteban, Rizzo \& Palau 2010; Umana et al. 2011b, 2012; Vamvatira-Nakou et al. 2013, 2015). These studies show that multiwavelength, high spatial resolution observations are needed to determine the mass-loss history and the geometry associated with massive stars near the end of their lives (Umana et al. 2011a). This information is fundamental to test evolutionary models. However, some of the parameters associated with the massloss still have large uncertainties, partly due to imprecise distance estimates, but also due to arbitrary assumptions about the nebula geometry.

To understand the importance of eruptive mass-loss in different metallicity environments, we observed at radio-wavelengths a subsample of LBVs in the Large Magellanic Cloud (LMC) that has a lower metal content $\left(Z<0.5 \mathrm{Z}_{\odot}\right)$ than the Milky Way. We selected this sub-sample based on the presence of an optical nebula (Weis 2003). In Agliozzo et al. (2012) (hereafter Paper I), we presented for the first time radio observations, performed with Australia Telescope Compact Array (ATCA) at 5.5 and $9 \mathrm{GHz}$. We detected the radio emission associated with LBVs RMC 127, RMC 143 and cLBVs S61 and S119. In this work, we present the most recent observations of cLBV S61, covering a larger spectral domain and including ATCA, Atacama Large Millimeter/Submillimeter Array (ALMA), and Very Large Telescope (VLT) VLT Imager and Spectrometer for mid Infrared (VISIR) data. The goals of this work are (i) to introduce a quantifiable and objective method for determining the nebular mass via Bayesian estimation of geometrical nebula parameters, (ii) to derive the mass-loss history with high temporal resolution, (iii) to compare the nebular properties of S61 with similar Galactic LBVNe, with respect to the nebular mass, kinematical age of the nebula, and dust production.

S61 (also named SK -67 266 and AL 418) is only a candidate LBV because since its first observations (Walborn 1977), it has not shown both spectroscopic and photometric variability. The star was classified as luminous supergiant (Ia) spectral type O8fpe. Originally, RMC 127 also belonged to this class, until it entered a state of outburst (between 1978 and 1980; Walborn 1982), during which the Of features disappeared and the spectrum evolved through an intermediate B-type to a peculiar supergiant A-type. In the meantime, Of-type emission was discovered during a visual minimum of the LBV AG Car (Stahl 1986), the Galactic twin of RMC 127. All these findings suggested that Ofpe stars and LBVs are physically related (e.g. Stahl 1986; Bohannan \& Walborn 1989; Smith et al. 1998) and Ofpe supergiant stars are now considered quiescent LBVs. In this paper, we will focus our attention on S61 for which Crowther \& Smith (1997) derived the following stellar parameters: $T_{\text {eff }}=27600 \mathrm{~K}, \log L / \mathrm{L}_{\odot}=5.76, \dot{M}=1.1 \times 10^{-5} \mathrm{M}_{\odot} \mathrm{yr}^{-1}$, and $v_{\infty}=250 \mathrm{~km} \mathrm{~s}^{-1}$. The paper is organized as follows: in Section 2, we present the new observations and data reduction; we describe the nebula around S61, its morphology, flux densities and spectral index (Section 3); we present our new public code RHocube (Nikutta \& Agliozzo 2016) to model 3D density distributions and derive via Bayesian inference the geometrical nebula parameters (Section 4). From the marginalized posteriors of all parameters obtained from fitting the 9 and $17 \mathrm{GHz}$ maps of S61, we estimate the posterior probability distribution (PDF) of the ionized mass contained in the nebula. In Section 5, we also show a method to derive the massloss history with high temporal resolution and we compare it with 
Table 1. Properties of the ATCA and ALMA maps.

\begin{tabular}{lcccccc}
\hline Array & $\begin{array}{c}v \\
(\mathrm{GHz})\end{array}$ & $\begin{array}{c}\text { HPBW } \\
(\operatorname{arcsec})\end{array}$ & $\begin{array}{c}\text { LAS } \\
(\operatorname{arcsec})\end{array}$ & $\begin{array}{c}\text { PA } \\
\left({ }^{\circ}\right)\end{array}$ & $\begin{array}{l}\text { Peak } \\
\left(\mathrm{mJy} \mathrm{beam}^{-1}\right)\end{array}$ \\
\hline ATCA & 17 & $0.836 \times 0.686$ & 6.5 & -10.6 & 0.142 & 0.016 \\
ATCA & 23 & $0.628 \times 0.514$ & 4.1 & -10.8 & 0.121 & 0.032 \\
ALMA & 343 & $1.23 \times 0.95$ & 6.7 & 78.6 & 0.290 & 0.072 \\
\hline
\end{tabular}

S61's empirical mass-loss rate. We discuss the derived extinction maps and interpret them together with the mid-IR and ALMA nondetections. Finally, in Section 6, we summarize our results.

\section{OBSERVATIONS}

\subsection{ATCA observations and data reduction}

We performed ATCA observations of S61 (together with two other Magellanic LBVs) between 2012 January 20 and 23. We used the array in the most extended configuration $(6 \mathrm{~km})$ and the Compact Array Broadband Backend (CABB) ' 15 mm' receiver in continuum mode. We split the receiver bandwidth in two 2-GHz sub-bands, one centred at $17 \mathrm{GHz}$ and the other at $23 \mathrm{GHz}$. This set-up was chosen in order to achieve enough spatial resolution to isolate possible contribution from the central source and also to obtain some spectral information. We observed the phase calibrator ICRF J052930.0-724528 for $1 \mathrm{~min}$, alternating with 7- or 10-min scans on target, depending on the weather. For the bandpass correction, we performed observations on the calibrator QSO J1924-2914 each day as well as observations of the flux calibrator ICRF J193925.0-634245. We also performed reference pointing frequently (about every $1-2 \mathrm{~h}$ ) to assure the pointing accuracy of the source observations. The total integration time obtained on each source was $8 \mathrm{~h}$.

We performed the data reduction and imaging using the MIRIAD package (Sault, Teuben \& Wright 1995). We split the data sets in two parts (one per central frequency) and reduced them separately. For the data editing, flagging, and calibration, we followed the standard calibration recipe for the millimetric band. We applied the opacity correction and flagged bad data, before calculating corrections for gains. We used observations of QSO J1924-2914, ICRF J193925.0-634245, and ICRF J052930.0-724528 for determining the bandpass, flux density, and complex gain solutions, respectively. Once corrected, the visibilities were inverted by Fourier transform. We chose the natural weighting scheme of the visibilities, for best sensitivity. Deconvolution of the dirty images was performed using the Clark algorithm (Clark 1980) and the selection of the clean components was done interactively. We then restored the clean components with the synthesized beam. Table 1 contains information about the synthesized beam (half-power beam width, HPBW) and position angle (PA), largest angular scale (LAS), peak flux densities, and rms-noise of the resulting images. At $17 \mathrm{GHz}$, we detect above $3 \sigma$ the nebular emission in its whole extension. At $23 \mathrm{GHz}$, the map is noisy because of the system response to bad weather at higher frequencies. For this reason, we do not show the $23 \mathrm{GHz}$ data. The radio map at $17 \mathrm{GHz}$ is illustrated in the upper-left panel of Fig. 1 .

We also include in our analysis the 5.5 and 9-GHz data from the ATCA observations performed in 2011 by means of the CABB '4cm-Band' (4-10.8 GHz) receiver. These data were presented in Paper I.
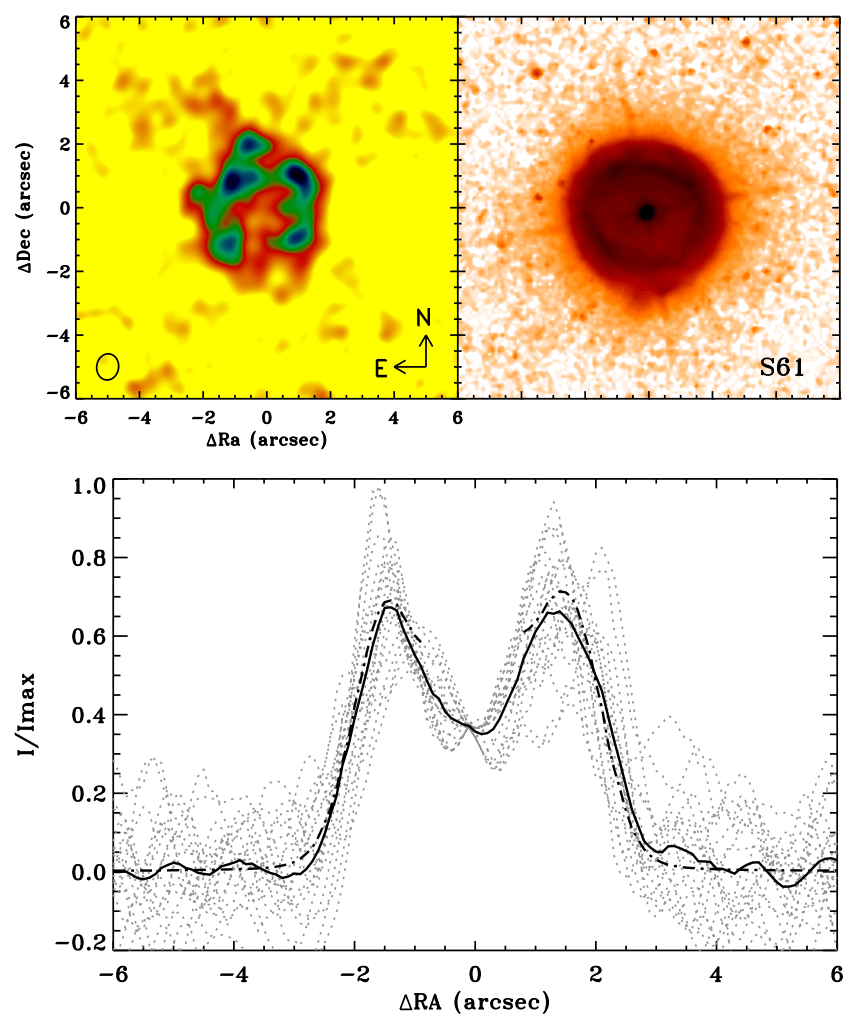

Figure 1. Upper-left panel: map of S61 at $17 \mathrm{GHz}$ obtained with the ATCA. The ellipse in the lower-left corner visualizes the synthesized beam. Upperright panel: archival $\mathrm{H} \alpha H S T$ image. Lower panel: surface brightness profiles. Grey dotted lines: profiles extracted from 18 cuts across the radio nebula, passing through the centre, in steps of $10^{\circ}$. Black line: mean of the grey dotted lines. Black dash-dotted line: mean surface brightness of the $\mathrm{H} \alpha$ image, derived from 18 cuts as explained before.

\subsection{The ALMA observation and data reduction}

S61 was observed as part of an ALMA Cycle-2 project studying three Magellanic LBVs (2013.1.00450.S, PI Agliozzo). A single execution of $80 \mathrm{~min}$ total duration, including the three targets, was performed on 2014-12-26 with $4012 \mathrm{~m}$ antennas, with projected baselines from 10 to $245 \mathrm{~m}$ and integration time per target of 16 min. A standard Band 7 continuum spectral setup was used, giving four 2-GHz width spectral windows of 128 channels of XX and YY polarization correlations centred at approximately 336.5 (LSB), 338.5 (LSB), 348.5 (USB), and 350.5 (USB) GHz. Online, antenna focus was calibrated during an immediately preceding execution and antenna pointing was calibrated on each calibrator source during the execution (all using Band 7). Scans at the science target tuning on bright quasar calibrators QSO J0538-4405 and Pictor A (PKS J0519-4546; an ALMA secondary flux calibrator 'grid' source) were used for interferometric bandpass and absolute flux scale calibration. Astronomical calibration of complex gain variation was made using scans on quasar calibrator QSO J0635-7516, interleaved with scans on the science targets approximately every six minutes. Of the 40 antennas in the array, 36 were fully used in the final reduction, with two more partially used due to issues in a subset of basebands and polarizations. Data were calibrated and imaged with the Common Astronomy Software Applications (CASA) package (McMullin et al. 2007).

Atmospheric conditions were marginal for the combination of frequency and necessarily high airmass (transit elevation $45^{\circ}$ for 


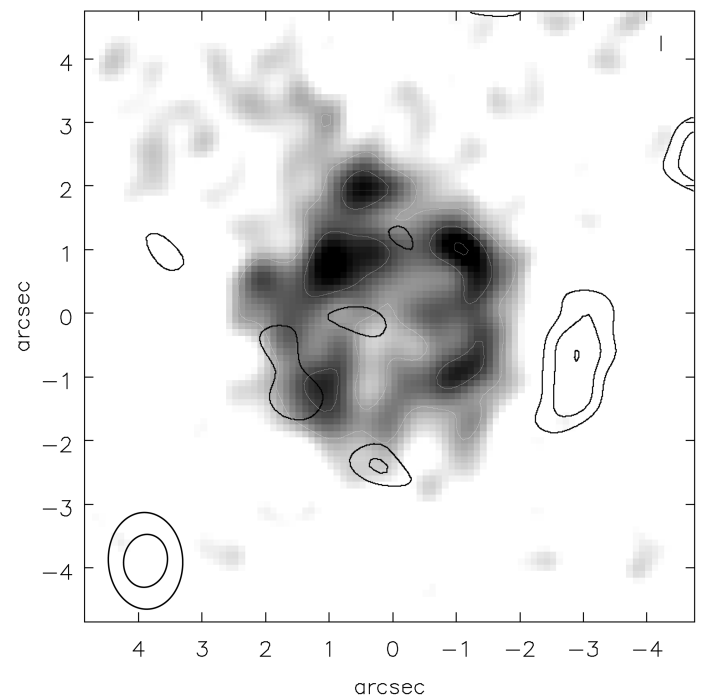

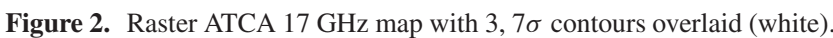
Black contours: from 2 to $4 \sigma$ levels in the ALMA map at $343 \mathrm{GHz}$. The big and small ellipses in the left bottom corner are, respectively, the ALMA and ATCA synthesized beams. ( $\mathrm{N}$ is up and $\mathrm{E}$ is left).

S61). Extra non-standard calibration steps were required to minimize image degradation due to phase smearing, to provide correct flux calibration, and to maximize sensitivity by allowing inclusion of shadowed antennas. As S61 was not detected, we defer discussion of these techniques to an article on the other sources in the sample (Agliozzo et al., submitted).

We derived the intensity image from naturally weighted visibilities to maximize sensitivity and image quality (minimize the impact of phase errors on the longer baselines). We imaged all spectral windows together $(343.5 \mathrm{GHz}$ average; approximately $7.5 \mathrm{GHz}$ usable bandwidth), yielding an rms noise of $72 \mu \mathrm{Jy}_{\text {beam }}{ }^{-1}$ in the image. This is compared to the proposed sensitivity of $40 \mu \mathrm{Jy} \mathrm{beam}^{-1}$, which could not be achieved as no further executions were possible during the appropriate array configuration in Cycles 2 and 3. With this sensitivity, we did not detect the nebula. In Fig. 2, we show the 2,3 , and $4 \sigma$ contours in the ALMA map (in black) on top of the ATCA $17 \mathrm{GHz}$ image. These contours do not have enough statistical significance. However, the elongated object west of the radio nebula has a peak at $4 \sigma$, but it is difficult to associate it with S61. Details of the ALMA map are listed in Table 1. Deeper observations with ALMA may detect the nebular dust and would certainly improve the constraints on the dust mass. This would be a good candidate for the potential 'high-sensitivity array' mode, combining all operational array elements (12 and $7 \mathrm{~m}$ antennas, typically at least 50 in total) in a single array with the 64-input Baseline correlator, when in the more compact $12 \mathrm{~m}$ array configurations (this may be offered from Cycle 6 in 2018).

\subsection{VLT/VISIR observations}

We proposed service-mode observations in the narrow-bandwidth filters PAH2_2 and Q1, centred, respectively, at 11.88 and $17.65 \mu \mathrm{m}$. The observations were carried out between 2015 September 3 and 4 . The observing mode was set for regular imaging, with pixel scale of 0.045 arcsec. The OBs were executed in conditions slightly worse (10 per cent) than specified in the scheduling constraints. Table 2 contains a summary of the VISIR observations.
Table 2. VISIR observational summary.

\begin{tabular}{lcccc}
\hline Date & Filter & Airmass & $\begin{array}{c}\text { DIMM Seeing } \\
(\operatorname{arcsec})\end{array}$ & $\begin{array}{c}\text { PWV } \\
(\mathrm{mm})\end{array}$ \\
\hline 2015-09-03 & PAH2_2 & 1.576 & 1.35 & 3.2 \\
2015-09-04 & Q1 & 1.590 & 1.38 & 1.8 \\
\hline
\end{tabular}

We have reduced the raw data by running the recipe visir_image_combine. $x m l$ of the VISIR pipeline kit (version 4.0.7) in the environment Esoreflex 2.8. We have compared the calibrator (HD026967 and HD012524) flux densities and sensitivities with the ones provided by the observatory from the same nights and found consistent results. Due to the non-detection of the science target, the data reduction pipeline has performed a straight combining of the images while correcting for jitter information from the fits headers, rather than stacking individual images with the shiftand-add strategy. In the last step, the pipeline has converted the final (combined) images from ADU to Jy pixel ${ }^{-1}$ by adopting the conversion factor derived from the calibrators. The output of the pipeline is a single image of $851 \times 851$ and $851 \times 508$ pixels in the PAH2_2 and Q1 filters, respectively. The two images are in units of Jy pixel ${ }^{-1}$. The rms-noise in the images is 0.08 and $2.1 \mathrm{mJy} \mathrm{pixel}^{-1}$, in the filters PAH2_2 and Q1, respectively, which translates in the noise of $\sim 40$ and $\sim 1000 \mathrm{mJy} \operatorname{arcsec}^{-2}$. We did not achieve the expected sensitivity (as estimated with the Exposure Time Calculator). This could be due to large-scale emission. We estimate that to detect a point-like source with a signal-to-noise ratio (SNR) of 3 , it should be at least $15 \mathrm{mJy}$ at $11.88 \mu \mathrm{m}$ and $430 \mathrm{mJy}$ at $17.65 \mu \mathrm{m}$.

\subsection{Optical data}

The $\mathrm{H} \alpha$ Hubble Space Telescope (HST) data (Weis 2003) were retrieved from the STScI data archive (proposal ID: 6540), as already described in Paper I. They were obtained with the Wide Field and Planetary Camera 2 (WFPC2) instrument using the $\mathrm{H} \alpha$-equivalent filter $\mathrm{F} 656 \mathrm{~N}$ and reduced by the standard HST pipeline. We combined the data set (four images with a $500 \mathrm{~s}$ exposure) following a standard procedure in IRAF to remove cosmic ray artefacts and to improve the SNR. We also recalibrated the HST image astrometrically using the Naval Observatory Merged Astrometric Dataset (NOMAD) catalogue (Zacharias et al. 2005) for a corrected overlay with the radio images. Finally, we converted the HST/WFPC2 image from counts pixel ${ }^{-1} \AA^{-1}$ units to erg cm $\mathrm{cm}^{-2} \mathrm{~s}^{-1}$ units, by multiplying with $2.9 \times 10^{-16} \times 21.5$ (where 21.5 is the F656N filter bandwidth in $\AA$ ).

\section{THE RADIO EMISSION}

In the upper panel of Fig. 1, the radio map (left) is compared with the H $\alpha H S T$ image (right). For a better visualization of the nebular morphology, we also show in the lower panel the radial surface brightness profiles, extracted from 18 cuts (grey dotted lines) across the radio nebula, passing through the centre and successively rotated by $10^{\circ}$. The black line is the arithmetic mean of the grey dotted lines. In a similar way, we derived the mean surface brightness of the H $\alpha$ HST image (black dash-dotted line), after convolving it with the radio beam. To block the emission of the central object in the $\mathrm{H} \alpha$ image, we applied a mask at the position of the star. The image shows that there is more substructure in the radio than in the optical, as is clearly evident by comparing the surface profiles. At $17 \mathrm{GHz}$, the nebula size is similar to the one in the $\mathrm{H} \alpha$ image. A two-colour 


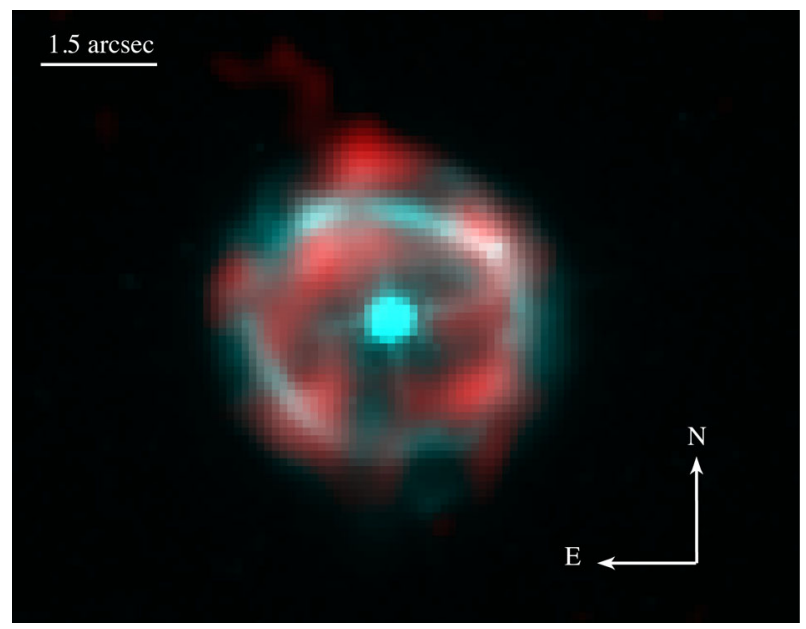

Figure 3. Two-colour image of S61. The red colour is the radio $17 \mathrm{GHz}$, the cyan one is the $\mathrm{H} \alpha$.

Table 3. Observed flux densities, angular sizes, and spectral index.

\begin{tabular}{ccccc}
\hline $\begin{array}{c}\mathrm{S}(5.5 \mathrm{GHz}) \\
(\mathrm{mJy})\end{array}$ & $\begin{array}{c}\mathrm{S}(9 \mathrm{GHz}) \\
(\mathrm{mJy})\end{array}$ & $\begin{array}{c}\mathrm{S}(17 \mathrm{GHz}) \\
(\mathrm{mJy})\end{array}$ & $\begin{array}{c}\text { Size } \\
(\operatorname{arcsec})\end{array}$ & $\langle\alpha\rangle$ \\
\hline $2.1 \pm 0.1$ & $2.2 \pm 0.3$ & $1.97 \pm 0.10$ & $4.5 \times 4.9$ & $-0.06 \pm 0.06$
\end{tabular}

image of the $17 \mathrm{GHz}$ and $\mathrm{H} \alpha$ data is shown in Fig. 3. In the northern part, apparently attached to the shell, there is a spur-emission, similar to G79.29+0.46 (e.g. Higgs, Wendker \& Landecker 1994). This compact object does not have a counterpart either at lower frequencies or in the optical. It might indicate an optically thick medium at the radio wavelengths.

Weis (2003) reported that the optical nebula of S61 is expanding spherically and with a velocity of $\sim 27 \mathrm{~km} \mathrm{~s}^{-1}$, although slightly redshifted to the west and blueshifted to the east, which they ascribed to a geometric distortion along the line of sight. The radio nebula at $17 \mathrm{GHz}$ is consistent with the shell geometry and therefore we will take it into account to model the radio emission (Section 4).

Table 3 lists the spatially integrated flux density and its associated error at $17 \mathrm{GHz}$, together with the estimated nebula angular sizes (not deconvolved by the synthesized beam). The integrated flux density was determined by using the CASA viewer. In particular, we selected with the polygonal tool the area above $3 \sigma$ level and integrated the emission over the nebula. The rms-noise in the map was evaluated in regions free of emission and hence flux density errors were estimated as $\epsilon=\sigma \sqrt{N}$, where $N$ is the number of independent beams in the selected region. Calibration flux density errors are usually negligible at these frequencies. The flux densities at 5.5 and $9 \mathrm{GHz}$ derived in Paper I are also shown in the table.

From the analysis of the spectral index, we can obtain information about the nature of the radio emission. We have computed the mean spectral index $\langle\alpha\rangle$ through a weighted fit of the power-law $S_{v} \propto v^{\alpha}$ between the flux densities at 5.5, 9, and $17 \mathrm{GHz}$ in Table 3 . The 'global' spectral index $\langle\alpha\rangle=-0.06 \pm 0.06$ is consistent with optically thin free-free emission. We have also obtained a spectral index map (per pixel). To this end, the highest resolution map $(17 \mathrm{GHz})$ was re-gridded and convolved with the beam at lower frequency. We show the spectral index map between 17 and $9 \mathrm{GHz}$ (with the beam about $1.5 \times 1.2 \operatorname{arcsec}^{2}$, as in Paper I) and its associated error map in Fig. 4. Since calibration errors are negligible, in the maps, the error in each pixel is mostly given by the sum in quadrature of the rms-noise in both the maps, in Jy pixel ${ }^{-1}$ units. In the inner part of the nebula, the mean spectral index is $\langle\alpha\rangle=-0.3 \pm$ 0.2 (where the error is the mean value in the error map), consistent with optically thin free-free emission. In the southern (bottom) part, we observe a higher spectral index $\left(\alpha_{\max }=0.8 \pm 0.3\right)$, suggesting some mechanism of self-absorption of the free-free emission, due to, for instance, density clumps. The spectral index analysis may be biased by the fact that the interferometer is sensitive to different large and intermediate angular scales at different frequencies. However, the LASs covered in the two data sets (12.9 and 6.5 arcsec at 9 and $17 \mathrm{GHz}$, respectively) are larger than the size of the nebula (Table 3 ). We also rely on a good $u v$ coverage at the intermediate angular scales acquired during the observations.

\section{MODELLING THE NEBULA}

In Paper I, we derived an estimate of the ionized mass in the S61 nebula from the $9 \mathrm{GHz}$ ATCA map. Simply, the total ionized mass can be estimated if the density of particles and the volume of the nebula are known. For non-self-absorbed optically thin free-free emission, the electron density $n_{\mathrm{e}}$ can be determined through the relation between the emission measure (EM),

$E M=\int_{0}^{s} n_{\mathrm{e}}^{2} \mathrm{~d} s\left[\mathrm{pc} \mathrm{cm}{ }^{-6}\right]$,

and the optical depth $\tau_{f f}(v)$ at frequency $v$

$\tau_{f f}(v)=8.24 \times 10^{-2}\left(\frac{T_{\mathrm{e}}}{\mathrm{K}}\right)^{-1.35}\left(\frac{v}{5 \mathrm{GHz}}\right)^{-2.1} \frac{E M}{\mathrm{pc} \mathrm{cm}^{-6}}$.

$\tau_{f f}(\nu)$ can be determined from the solution of the radiative transfer equation $\left(B_{v}=B_{b b}(T) \tau_{f f}(v)\right)$ by setting as $B_{v}$ the radio brightness and by assuming a blackbody with temperature $T$ equal to the electron temperature $T_{\mathrm{e}}$. Therefore, in Paper I, we derived an average $n_{\mathrm{e}}$ from the mean $E M$ (integrated over the nebula) and assumed as $s$ the transversal size of the nebula (measured on the radio map). With these values, we estimated for S61's nebula an ionized mass of $\sim 0.8 \mathrm{M}_{\odot}$. In reality, $n_{\mathrm{e}}$ may vary inside the nebula. Furthermore, the geometrical depth $s$ may vary for different line of sights and then requires a proper geometrical model. Therefore, we propose a new approach to fit all the pixels of the radio maps with a global geometrical 3D density model of the nebula. Obviously, the nebula has to be spatially resolved. Instrumental effects on the nebula size due to bad resolution have to be negligible. If not, the estimated mass may be inaccurate.

\subsection{D density model RHOCUBE}

We have written and make publicly available ${ }^{1}$ RHocuBE (Nikutta \& Agliozzo 2016), a PYTHON code to model 3D density distributions $\varrho(x, y, z)$ on a discrete Cartesian grid and their integrated 2D maps $\int \mathrm{d} z \varrho(x, y, z)$. It can be used for a range of applications; here we model with it the electron number density $n_{e}(x, y, z)$ in LBV shells and from this compute the emission measure, $E M$, given in equation (1).

The code repository includes several useful 3D density distributions, implemented as simple PYTHON classes, e.g. a power-law shell (PLS), a truncated Gaussian shell, a constant-density torus, dual cones, and also classes for spiralling helical tubes. Other distributions can be easily added by the user. Convenient methods for

\footnotetext{
${ }^{1}$ https://github.com/rnikutta/rhocube
} 

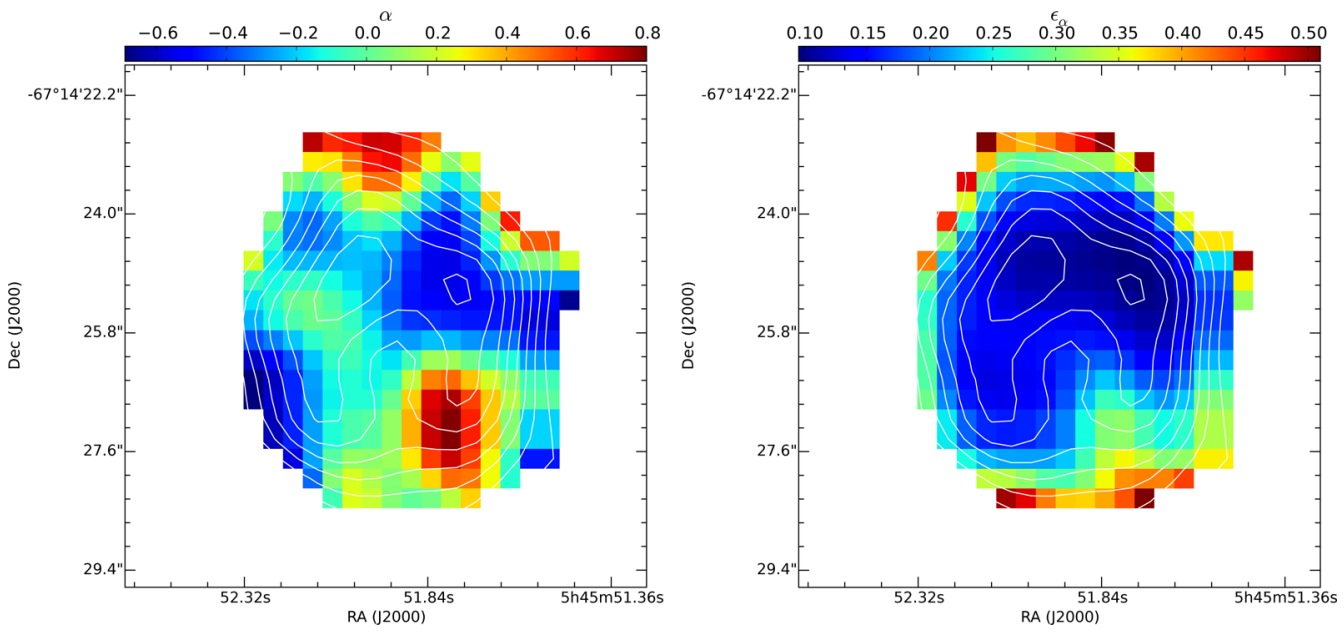

Figure 4. Left: spectral index map between 9 and $17 \mathrm{GHz}$. Right: spectral index error map. Before the computation, the map at $17 \mathrm{GHz}$ was reconvolved to match the beam at $9 \mathrm{GHz}$. The white contours indicate flux densities above $3 \sigma$ (at integer steps) of the $17 \mathrm{GHz}$ emission at the $9 \mathrm{GHz}$ resolution.

shifts and rotations in 3D are also provided. If necessary, an arbitrary number of density distributions can be combined into the same model cube and the integration $\int \mathrm{d} z$ will be correctly performed through the joint density field. Please see Appendix A4 and the code repository for usage examples of RHOCUBE and for details of the implementation.

\subsection{Bayesian parameter inference}

We will apply RHOcuBE to our problem of estimating the physical parameters of the observed emission maps by modelling the underlying 3D electron density distribution $n_{\mathrm{e}}(x, y, z)$. We employ a Bayesian approach and compute marginalized posterior density distributions of model parameters from the converged chains of Markov Chain Monte Carlo (MCMC) runs. Bayes' theorem and the details of MCMC sampling are described in Appendix B. Our routines to fit RHOCUBE models to data are available as supplemental materials, in Section 7.

We begin with any 3D model for the geometry of the nebula, e.g. a truncated Gaussian shell. At every sampling step of the MCMC procedure, we draw a random vector $\boldsymbol{\theta}$ of values for the free model parameters (e.g. the shell radius, width, lower and upper truncation radii, and $x$ and $y$ offsets). We then compute the 3D electron density distribution $n_{\mathrm{e}}(x, y, z \mid \boldsymbol{\theta})$ and from it the squared integrated 2D map $E M_{\text {mod }}=\int n_{\mathrm{e}}^{2}(x, y) \mathrm{d} z$. The normalization of $n_{\mathrm{e}}$ is at first arbitrary, but by comparison with the observed map $E M_{\text {obs }}$, we can find a global scale $S$ such that the likelihood is maximized, or equivalently, the chi-squared statistic $\chi^{2}=\sum_{i}\left(d_{i}-S m_{i}\right)^{2} / \sigma_{i}^{2}$ is minimized. The $d_{i}$ are the measured pixel values $E M_{\mathrm{obs}}^{i}$ (for unmasked pixels only) and $m_{i}$ their modelled counterparts. $S$ can be computed analytically (e.g. Nikutta 2012). Note that the pixels $i$ are independent.

\subsubsection{Application to the S61 nebula}

In the following, we apply RHocuBe together with MCMC and Bayesian inference to fit the EM maps obtained for S61 from the data at 9 and $17 \mathrm{GHz}$. Note that the angular resolution in the map at $9 \mathrm{GHz}$ is poor and affects the nebula size (Paper I). We only use
Table 4. Uniform priors adopted for the truncated normal shell and the PLS models. The priors were chosen from visual inspection of the 9 and $17 \mathrm{GHz}$ maps and were limited to meaningful ranges.

\begin{tabular}{lccc}
\hline Parameter & Units & $9 \mathrm{GHz}$ & $17 \mathrm{GHz}$ \\
\hline Truncated normal shell & & & \\
$r$ & $\mathrm{pc}$ & {$[0,0.3]$} & {$[0,0.3]$} \\
$\sigma_{\mathrm{r}}$ & $\mathrm{pc}$ & {$[0,0.7-r]$} & {$[0,0.6-r]$} \\
$r_{\text {lo }}$ & $\mathrm{pc}$ & {$[0, r]$} & {$[0, r]$} \\
$r_{\text {up }}$ & $\mathrm{pc}$ & {$\left[0, r+\sigma_{\mathrm{r}}\right]$} & {$\left[0, r+\sigma_{\mathrm{r}}\right]$} \\
Power-law shell & & & \\
$r_{\text {in }}$ & $\mathrm{pc}$ & {$[0,0.3]$} & {$[0,0.3]$} \\
$r_{\text {out }}$ & $\mathrm{pc}$ & {$\left[r_{\text {in }}, 0.7\right]$} & {$\left[r_{\text {out }}, 0.6\right]$} \\
\hline
\end{tabular}

these data to test our procedure and to compare the mean $E M_{\text {mod }}$ and the derived ionized mass with those estimated in Paper I.

The radio maps of S61, while irregular, are indicative of a spherical matter distribution (e.g. Fig. 1, top-left). This is even more apparent in the $\mathrm{H} \alpha$ map (Fig. 1, top-right; see also Pasquali, Nota \& Clampin 1999; Weis 2003). We therefore modelled with RHOCUBE the electron density distribution by using the following geometries: a truncated Gaussian (normal) shell and a PLS. For the truncated normal shell (hereafter TNS), the free parameters are six: the shell radius $\mathrm{r}$, the width $\sigma_{\mathrm{r}}$ of the Gaussian around $r$, the lower and upper clip radii $r_{\text {lo }}$ and $r_{\text {up }}$, and finally we allow for minute offsets in the plane of the sky, xoff and yoff, to account for possible de-centring of the observed shell. For the PLS, the free parameters are four: the inner and outer radii $r_{\text {in }}$ and $r_{\text {out }}$, xoff, and yoff. For the PLS geometry, we explored the cases with exponents: 0 (i.e. constant density shell), -1 , and -2 . We noticed that exponents smaller than -2 were producing lower quality results and therefore we will not comment on them. We used uniform prior probability distributions (i.e. before introducing the model to the observed data) on the shell radius, width, and both clip radii for the TNS geometry and for the inner and outer radii for the PLS geometry.

The ranges of these parameters must, of course, be limited to meaningful values. For convenience, we converted the $x$ and $y$ pixel units in the maps from arcseconds to parsecs, assuming a distance of $48.5 \mathrm{kpc}$. Hence, from visual inspection of the map, we adopted the values listed in Table 4. As priors of both offsets xoff and yoff, 
Table 5. Inference of model parameters, as derived from data at 9 and $17 \mathrm{GHz}$. A truncated normal spherical shell was used as a model. MAP $=$ maximum-a-posteriori values. Median $=$ median of marginalized posterior distributions, with $1 \sigma$ confidence intervals. Note that $M_{\text {ion }}$ is a derived quantity, i.e. not a free (modelled) parameter.

\begin{tabular}{|c|c|c|c|c|c|}
\hline \multirow[t]{3}{*}{ Parameter } & \multirow[t]{3}{*}{ Units } & \multicolumn{4}{|c|}{ Truncated normal shell } \\
\hline & & \multicolumn{2}{|c|}{ MAP } & \multicolumn{2}{|c|}{ Median } \\
\hline & & $\begin{array}{r}9 \mathrm{GHz} \\
\left(\chi_{\mathrm{r}}^{2}=46\right)\end{array}$ & $\begin{array}{r}17 \mathrm{GHz} \\
\left(\chi_{\mathrm{r}}^{2}=33\right)\end{array}$ & $9 \mathrm{GHz}$ & $17 \mathrm{GHz}$ \\
\hline$r$ & $\mathrm{pc}$ & 0.19 & 0.30 & $0.19_{-0.09}^{+0.07}$ & $0.18_{-0.09}^{+0.08}$ \\
\hline$\sigma_{\mathrm{r}}$ & $\mathrm{pc}$ & 0.50 & 0.30 & $0.35_{-0.15}^{+0.13}$ & $0.29_{-0.13}^{+0.12}$ \\
\hline$r_{\mathrm{lo}}$ & $\mathrm{pc}$ & 0.05 & 0.28 & $0.08_{-0.06}^{+0.09}$ & $0.07_{-0.05}^{+0.09}$ \\
\hline$r_{\text {up }}$ & $\mathrm{pc}$ & 0.67 & 0.58 & $0.35_{-0.12}^{+0.15}$ & $0.31_{-0.12}^{+0.13}$ \\
\hline$x$-offset & $\mathrm{pc}$ & -0.07 & -0.04 & $0.00_{-0.10}^{+0.09}$ & $0.00_{-0.03}^{+0.03}$ \\
\hline$y$-offset & $\mathrm{pc}$ & -0.08 & -0.01 & $0.00_{-0.10}^{+0.09}$ & $-0.00_{-0.03}^{+0.03}$ \\
\hline$M_{\text {ion }}$ & $\mathrm{M}_{\odot}$ & 0.29 & 0.13 & $0.07_{-0.04}^{+0.09}$ & $0.03_{-0.02}^{+0.04}$ \\
\hline
\end{tabular}

we adopted very narrow Gaussians, truncated at \pm 2 pixels from the central pixel. The maps supplied to the code are $41 \times 41$ and 101 $\times 101$ pixels (for the $9 \mathrm{GHz}$ and $17 \mathrm{GHz}$ data, respectively). In Tables 5 and 6, we show the resulting marginalized posteriors from the geometries mentioned before.

Fig. 5 illustrates only the posteriors from the fit of the 9 and $17 \mathrm{GHz}$ data with a truncated Gaussian shell. These posteriors were obtained after drawing $2 \times 10^{4} \mathrm{MCMC}$ samples. Many fewer samples are necessary for convergence $(\sim 1000$ may be sufficient), but more samples produce smoother histograms. In the figure, we do not show the posteriors for the offsets xoff and yoff that are very narrow and centred, i.e. the shell is not significantly shifted from the central pixel. The MCMC chain histograms are shown in red, a Gaussian kernel density estimation is overplotted in black. ${ }^{2}$ Blue dashed vertical lines indicate the single best-fitting values (maximum-a-posteriori, MAP) of the MCMC chains, i.e. the combination of parameter values which simultaneously maximize the likelihood. Note that this need not be the 'most typical' solution. Green dotted lines mark the median values of the marginalized posterior PDFs. These statistics, and the $1 \sigma$ confidence intervals around the median, are summarized in Table 5. Note that for the $9 \mathrm{GHz}$ data, PLS (with exp $=0$ ) equally produces a good-quality result. For the $17 \mathrm{GHz}$ data, the PLS (with exp $=0$ ) produces the best fit. The MAP models (for unmasked pixels) have a formal $\chi_{\mathrm{r}}^{2}$ as shown in the tables. While large values, considering the simple model and the clearly not entirely spherical/symmetric EM map, are acceptable. In the figure, we only show our favourite models chosen because they produce more agreeable distributions and radial profiles. For this reason, we now describe only them.

The distribution of shell radii (panel 2 in Fig. 5) is quite broad, but clearly peaks within the shell. The radial thickness of the Gaussian shell is symmetric around the peak in panel 3. A similar comment can be given for the upper clip radius $r_{\text {up }}$ (panels 5). The lower clip radius $r_{\mathrm{lo}}$ (panels 4 ) is left-bounded at 0 and the decline at the large-values tail is driven by our prior requirement. The observed $9 \mathrm{GHz}$ and $17 \mathrm{GHz} E M_{\mathrm{obs}}$ maps are shown in panel (0) and the model shell corresponding to the MAP model is shown in (1). This panel also shows with solid, dashed, and dotted circles the medianmodel values of $r, r \pm \sigma_{\mathrm{r}}$, and $r_{\mathrm{up}}$, respectively. Panel (7) illustrates

${ }^{2}$ Computed with SEABORN, available from http://stanford.edu/mwaskom/ software/seaborn

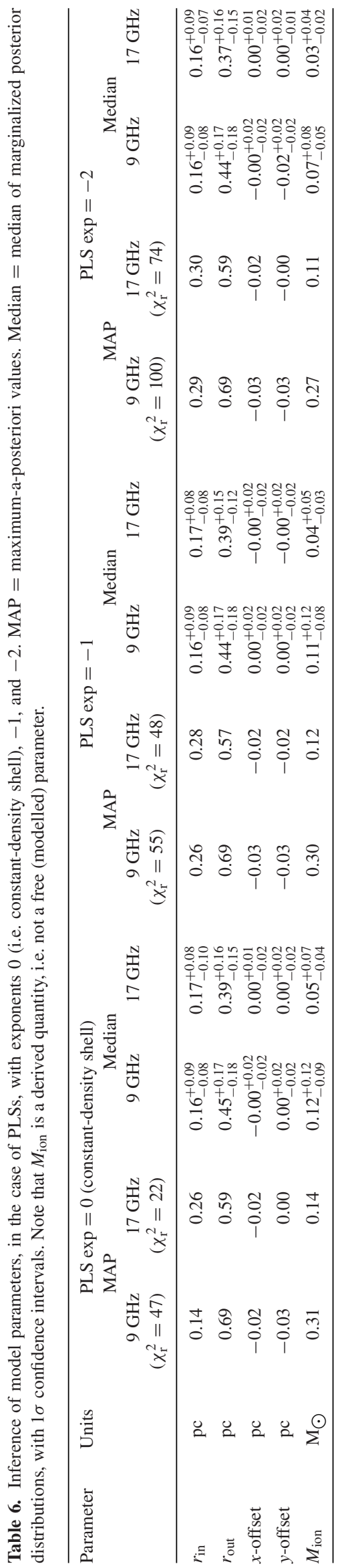



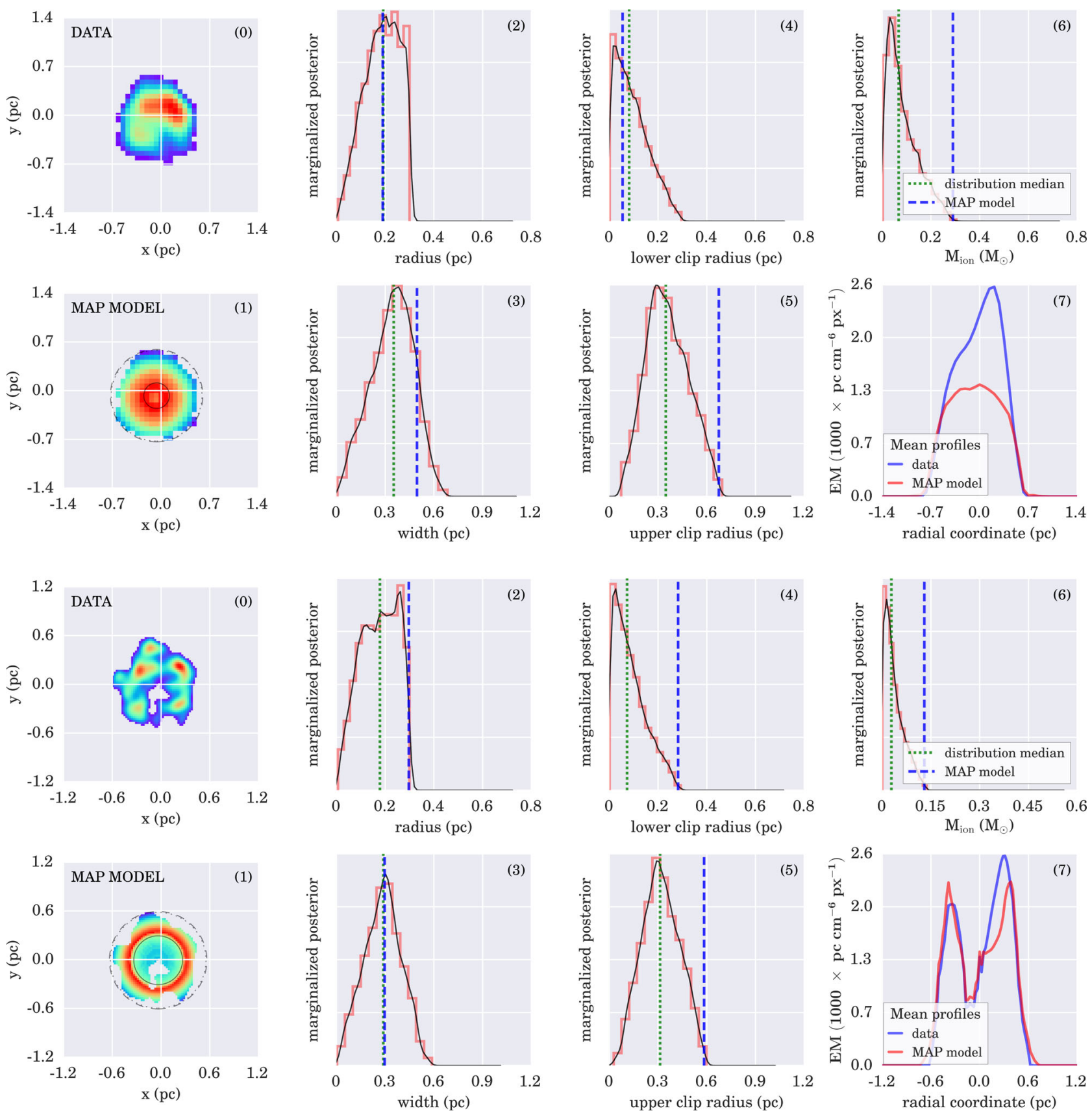

Figure 5. Results of fitting the $9 \mathrm{GHz}$ (top two rows) and $17 \mathrm{GHz}$ (bottom two rows) EM maps of $\mathrm{S} 61$ with truncated Gaussian shells. Panel (0) shows the data with linear sizes relative to the central pixel, with a $3 \sigma$ mask applied. Panel (1) shows the MAP model. Indicated as solid, dashed, and dotted circles are the median values of $r, r \pm \sigma_{\mathrm{r}}$, and $r_{\text {up }}$, respectively. Panels (2)-(6) show as red histogram the posteriors of four model parameters and of the derived ionized mass $M_{\text {ion. }}$. All histograms are normalized to unit area. The smooth black curve is a Gaussian kernel density estimation. The vertical blue dashed and green dotted lines indicate the maximum-a-posteriori (MAP) and median value of the MCMC chain, respectively. The blue dashed line in panel (6) locates the ionized mass value of the model shown in panel (1). Panel (7) illustrates azimuthal mean profiles derived from 18 extracted cuts along the EM (data: blue line; model: red line).

azimuthal mean profiles derived from 18 extracted cuts along $E M_{\mathrm{obs}}$ (blue line) and $E M_{\mathrm{MAP}}$ (red line). While the MAP model for the $9 \mathrm{GHz}$ seems underestimated, the $17 \mathrm{GHz}$ model profile is quite satisfactory.

As evident in the tables, different models (TNS, PLS exp $=0$, PLS $\exp =-1$ ) produce similar-quality results, meaning that with the current data, we cannot constrain the electron density distribution in the S61 nebula.

\subsection{Ionized mass}

Knowing the 3D distribution of the electron number density $n_{\mathrm{e}}(x$, $y, z)$, we can now compute the total ionized mass contained in the shell via

$\mathrm{M}_{\mathrm{ion}}=\frac{m_{p}}{\mathrm{M}_{\odot}} \int \mathrm{d} V n_{\mathrm{e}}(x, y, z)$, 
with $m_{\mathrm{p}}$ and $\mathrm{M}_{\odot}$ the proton and solar masses, under the assumption that the gas comprises only ionized hydrogen. For simple symmetric geometries and density distributions, this integral can be evaluated analytically (e.g. a constant-density shell), but it might be significantly more challenging for more complicated geometries and more complex density fields. In our discretized 3D Cartesian grid, realizing that the volume of a $3 \mathrm{D}$-voxel is $(\Delta x)^{3}$, because $\Delta x=\Delta y=$ $\Delta z$, equation (3) simplifies to

$\mathrm{M}_{\mathrm{ion}}=\frac{m_{\mathrm{p}}}{\mathrm{M}_{\odot}}(\Delta x)^{3} \sum_{i} n_{\mathrm{e}}\left(x_{i}, y_{i}, z_{i}\right)$,

where the index $i$ runs over all voxels (recall that they are independent).

Thanks to the MCMC approach, we can use the entire converged chains of model parameter values to compute posterior distributions of derived quantities (i.e. not modelled quantities), such as the ionized mass here. The resulting marginalized posterior distribution for the TNS geometry is shown in panel (6) of Fig. 5, with the purpose to provide an example. In fact, as mentioned before, we do not have a statistically strong model to discern among the possible density distributions. However, it is comforting to see that all the models produce similar masses (see Tables 5 and 6).

The TNS model of the $9-\mathrm{GHz}(17-\mathrm{GHz})$ data generates a peaked and skewed distribution of $M_{\text {ion }}$, with median $0.07_{-0.04}^{+0.09} \mathrm{M}_{\odot}$ $\left(0.03_{-0.02}^{+0.04} \mathrm{M}_{\odot}\right)$. The MAP-model values are 0.29 and $0.13 \mathrm{M}_{\odot}$. These are located always in the right side of the distribution, probably due to our prior requirements to fit the shell within the edge of the nebula, rather than the edge of the image frame. We remind the reader that the modelling of the $9 \mathrm{GHz}$ data was proposed in order to test the code and to compare the results with our previous estimation $\left(\sim 0.8 \mathrm{M}_{\odot}\right.$; Paper I). The value derived with this new approach is about 2.7 times smaller than the previous estimate. However, because of the asymmetry of the nebula at $9 \mathrm{GHz}$, the model seems to underestimate, on average, $E M_{\text {obs }}$ (see panel 7 in Fig. 5). According to this, the two methods may not disagree each other. The advantage of the proposed new method is that it requires no assumptions about the nebula depth $s$.

The derived mass from the fits of the $17 \mathrm{GHz}$ data $\left(0.11-0.14 \mathrm{M}_{\odot}\right)$ are more representative, because of the smaller $\chi_{\mathrm{r}}^{2}$ than the $9 \mathrm{GHz}$ data. The mean profile of $E M_{\mathrm{MAP}}$ reproduces satisfactorily $E M_{\mathrm{obs}}$ (see bottom panel 7 in Fig. 5). More importantly, the angular resolution achieved at $9 \mathrm{GHz}$ affects the nebular size, resulting in a larger volume to model. For further analysis, we will then adopt the MAP model from the fit of the $17 \mathrm{GHz}$ data.

The mass estimated here are at least an order of magnitude smaller than the one derived by Pasquali et al. (1999) from the $\mathrm{H} \alpha$ luminosity and from optical emission lines. The discrepancy may be due to a combination of different assumptions and methods. For instance, Pasquali et al. (1999) assumed for the LMC a distance of $51.2 \mathrm{kpc}$ and measured a size for the nebula of $7.3 \operatorname{arcsec}$ (i.e. $1.8 \mathrm{pc}$ ) from an image with a poorer resolution. Their estimation of the average electron density was also uncertain due to uncertainties of the [S II] $6717 / 6731$ ratio. It would be interesting to compare our results with integral field unit observations of the nebula around S61.

As shown in Section 3, there are hints of inhomogeneities in the nebula. Following Abbott, Bieging \& Churchwell (1981), who described the radio spectrum of a clumped stellar wind, we can assume discrete gas clumps of relatively higher density $\left(n_{\mathrm{H}}\right)$, embedded in a lower density medium $\left(n_{\mathrm{L}}\right)$. Both the clumps and the inter-clump medium are assumed optically thin. The clumps are distributed randomly throughout the volume of the nebula. If we define $f$ as the fractional volume which contains material at density $n_{\mathrm{H}}$, then equation (1) becomes

$E M=\int_{0}^{s}\left(f n_{\mathrm{H}}^{2}+(1-f) n_{\mathrm{L}}^{2}\right) \mathrm{d} s$.

The ionized mass in the nebula can be underestimated if not corrected for clumpiness. In fact, in the simple case of an empty inter-clump medium $\left(n_{L}=0\right)$, it is possible to demonstrate that $M \propto S_{v}^{1 / 2} f^{-1 / 2}$. For a filling factor $f=0.5$, the ionized mass would be about 41 per cent larger than in the case of a homogeneous nebula. For a more generic case, the factor to correct the estimated ionized mass is $\left(f n_{\mathrm{H}}^{2}+(1-f) n_{\mathrm{L}}^{2}\right)^{1 / 2}$. The ionized mass could also be underestimated in the emitting regions with a positive spectral index. In the specific case of S61, where $\alpha \sim 0.8$, the optical depth is $\lesssim 1$. Moreover, the region with positive $\alpha$ is small. We can assume that the underestimation of mass is negligible.

By using our model, we can derive the ionizing photon flux as

$F_{U V}=(\Delta x)^{3} \beta_{2} \sum_{i} n_{\mathrm{e}}^{2}\left(x_{i}, y_{i}, z_{i}\right)$,

with $\beta_{2}$ the recombination coefficient of the second energy level of $\mathrm{H}$. It is still not known if the nebula is density or ionization bounded, therefore we have to keep in mind that this could be a lower limit. We find $\log \left(F_{\mathrm{UV}}\right)=44.5$, which corresponds to a supergiant of spectral type later than B3. This is too cold compared with S61's star (Crowther \& Smith 1997; Pasquali et al. 1999). Note that the recombination time for such a nebula would be typically of some thousands years, implying that LBV variability from the ionizing source would be negligible. This may indicate that the nebula is density-bounded and that part of the stellar UV flux escapes from the nebula.

\section{DISCUSSION}

\subsection{The mass-loss history}

Starting from our best model in Section 4, we now derive the massloss history of S61 with high temporal resolution, keeping in mind that we could not constrain the electron density distribution.

If we know the expansion velocity of this nebula, each voxel of our data cube corresponds to a kinematical age. For instance, in the case of S61, the expansion velocity is $27 \mathrm{~km} \mathrm{~s}^{-1}$ (Weis 2003) and each voxel in the model of the $17 \mathrm{GHz}$ data has a $1 \mathrm{D}$ size equal to $0.1 \mathrm{arcsec}$, which, at the assumed distance of the LMC (48.5 kpc), corresponds to $7.3 \times 10^{11} \mathrm{~km}$ and therefore to a kinematical interval of $\sim 850 \mathrm{yr}$. We know the mass in each voxel and therefore we can derive the average mass-loss rate $\mathrm{d} M / \mathrm{d} t$ in intervals of $850 \mathrm{yr}$. According to the shell geometry adopted for the S61 nebula, we can assume that the star has lost mass isotropically. We can finally integrate $\mathrm{d} M / \mathrm{d} t$ over shells of radius $r$ and thickness $d r$, where $r$ can vary between 0 and $\frac{N}{2}+1(N$ is the number of pixels in each dimension of the cube). The resulting mass-loss rates for S61 are shown in Fig. 6. In this particular example, the peak of the mass-loss has occurred at epoch $\sim 19100 \mathrm{yr}$ with a rate of $1.5 \times$ $10^{-5} \mathrm{M}_{\odot} \mathrm{yr}^{-1}$. However, we note that the finite resolution due to the synthesized beam may mean that the real distribution is less smooth.

The mass-loss rates derived in Fig. 6 are consistent with our non-detection of the stellar wind in the radio maps. If we assume a spherical mass-loss for the star and then the model in Panagia \& Felli (1975), with a terminal velocity of $250 \mathrm{~km} \mathrm{~s}^{-1}$ (Crowther \& Smith 1997), an electron temperature of $6120 \mathrm{~K}$ 


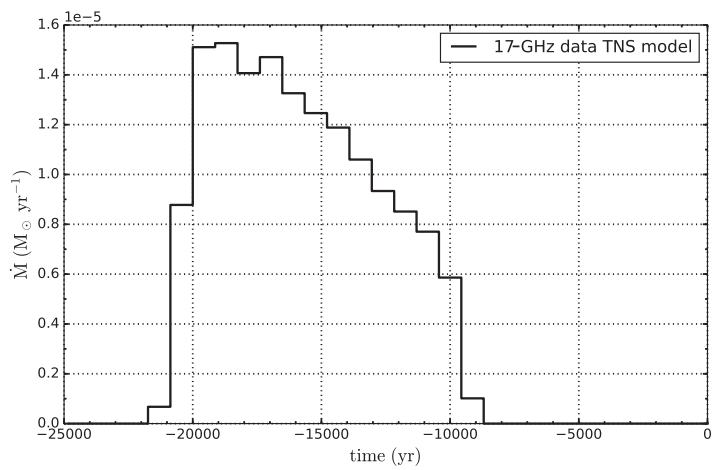

Figure 6. Mass-loss rate of S61 as derived from fitting the circumstellar nebula. Each bin of the histogram corresponds to $\sim 850 \mathrm{yr}$. The plot does not contain information about the present-day mass-loss rate.

(Pasquali et al. 1999) and a flux density equal to three times the noise in the map at $17 \mathrm{GHz}$, we derive a $3 \sigma$ upper limit of $\sim 2.3$ $\times 10^{-5} \mathrm{M}_{\odot} \mathrm{yr}^{-1}$, for a fully ionized wind with solar abundances. This upper limit is consistent with the mass-loss rate of $\sim 1.1 \times$ $10^{-5} \mathrm{M}_{\odot} \mathrm{yr}^{-1}$ derived by Crowther \& Smith (1997), which would be within the distribution in Fig. 6 . The value by Pasquali et al. (1997) of $\sim 2.2 \times 10^{-5} \mathrm{M}_{\odot} \mathrm{yr}^{-1}$ derived from $\mathrm{H}$ emission lines seems inconsistent with the radio non-detection.

We now compare the mass-loss history with the empirical massloss rate, as predicted for $\mathrm{O}, \mathrm{B}$ normal supergiants following the procedure described in Vink, de Koter \& Lamers (2000). We first assume the stellar parameters by Crowther \& Smith (1997) $\left(T_{\text {eff }}=27.6 \mathrm{kK}, \log \left(\frac{L}{\mathrm{~L} \odot}\right)=5.76\right.$, and $\left.v_{\infty}=250 \mathrm{~km} \mathrm{~s}^{-1}\right)$, a metallicity of $Z=0.5 \mathrm{Z}_{\odot}$, and an initial stellar mass of $\sim 60 \mathrm{M}_{\odot}$ (according to the evolutionary tracks by Schaller et al. 1992). The small nebular mass derived in the previous section suggests that the star has a stellar mass similar to its initial value and then the mass-loss rate is comparable to the one relative to the $\mathrm{O}, \mathrm{B}$ main-sequence stars (the reduced stellar mass of LBVs causes a strong increase in the mass-loss rate with respect to normal O, B supergiants, as showed by Vink \& de Koter 2002). The empirical mass-loss rate derived with the mentioned parameters is $6.6 \times 10^{-6} \mathrm{M}_{\odot} \mathrm{yr}^{-1}$, which is close to the average value of the distribution in Fig. 6 . The consequence of this result is either that the mass-loss occurred with a constant wind (constant density model) or the mass-loss rates varied due to excursion through the bi-stability jump (power-law electron density model). In this latter case, the bi-stability jump for S61 would occur at $T_{\text {eff }} \sim 23.5 \mathrm{kK}$, with a mass-loss rate of $3.3 \times 10^{-5} \mathrm{M}_{\odot} \mathrm{yr}^{-1}$, which is consistent with the peak in Fig. 6 within a factor of $\sim 2$. In both cases, we can probably exclude that S61 lost mass through eruptions, as normal stellar winds perfectly explain the observations. If we instead use the stellar parameters by Pasquali et al. (1997) $\left(T_{\text {eff }}=36.1 \mathrm{kK}, \log \left(\frac{L}{\mathrm{~L}_{\odot}}\right)=6.1\right)$, the derived empirical mass-loss rate is $7.0 \times 10^{-5} \mathrm{M}_{\odot} \mathrm{yr}^{-1}$, which is far higher than our observational values derived in the previous section.

\subsection{Extinction map and nebular dust}

We have derived the extinction map of S61 by comparing pixelby-pixel the highest resolution radio image (17 GHz) with the HST $\mathrm{H} \alpha$ image, as the two emissions trace the same gas (Paper I). According to Pottasch (1984), if the optical $\mathrm{H} \alpha$ emission is due to the de-excitation of the recombined $\mathrm{H}$ atom and the radio continuum

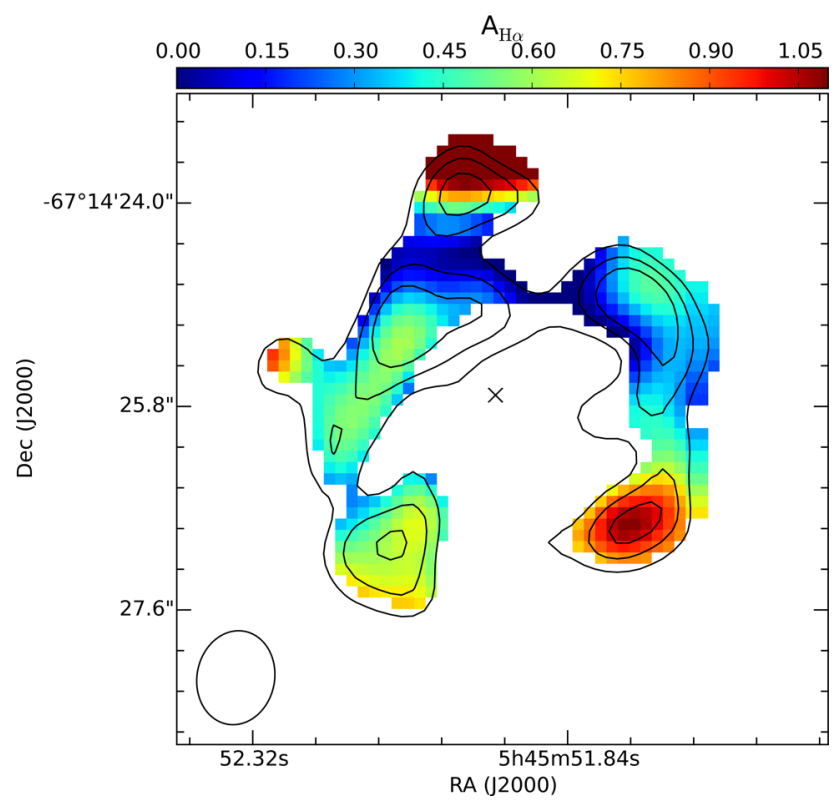

Figure 7. Extinction map in $\mathrm{H} \alpha$, as derived by comparing the $\mathrm{H} \alpha$ recombination line and the centimetre $(17 \mathrm{GHz})$ emission above $5 \sigma$. The central star was masked with a circular aperture in the optical image. The black contours are 5,7 , and $9 \sigma$ levels of the radio emission and the black ellipse is the resolution. The black cross represents the position of the star, according to the SIMBAD data base.

emission to free-free encounters, one can determine the extinction of the optical line by comparing the two brightnesses, as

$F_{v(\text { expected })}=2.51 \times 10^{7} T_{\mathrm{e}}^{0.53} v^{-0.1} Y F_{\mathrm{H}_{\beta}} \quad[\mathrm{Jy}]$,

where $T_{\mathrm{e}}$ is the electron temperature of the nebula in units of $\mathrm{K}, v$ is the radio frequency in $\mathrm{GHz}, \mathrm{Y}$ is a factor incorporating the ionized $\mathrm{He} / \mathrm{H}$ ratios (assumed to be 1 , as in Paper I), and

$F_{\mathrm{H}_{\beta}}=\frac{1}{2.859}\left(\frac{T_{\mathrm{e}}}{10^{4}}\right)^{0.07} F_{\mathrm{H}_{\alpha}}$

for the theoretical Balmer decrement.

We re-gridded the HST image to the same grid of the radio map and converted it to Jy pixel $^{-1}$ unit. We convolved the optical image with the radio beam (elliptical Gaussian with HPBW as in Table 1). Adopting as electron temperature 6120 K (Pasquali et al. 1999), we derive the expected radio map from the $\mathrm{H} \alpha$ recombination-line emission. Keeping in mind that we want to estimate the expected free-free emission from the optical line in the nebula, we masked the $\mathrm{H} \alpha$ emission from the star. Finally, the extinction map in $\mathrm{H} \alpha$

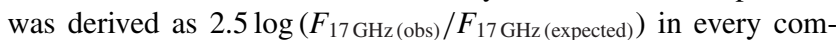
mon pixel with brightness above $5 \sigma$, where $\sigma$ was computed by summing in quadrature the noise in the maps and calibration uncertainties (however negligible). As a result of this procedure, we obtained the extinction map illustrated in Fig. 7. Small extinction due to dust is evident across the whole region. The range of values for $A_{\mathrm{H} \alpha}$ across the nebula is between $\sim 0.1$ and 1.09 . The maximum value for $A_{\mathrm{H} \alpha}$ is 1.8 and corresponds to the spur-object in the northern (upper) part.

To derive a range of possible characteristic temperatures for the dust that extinguishes the optical emission, we fitted the flux density distribution from the mid- to the far-IR. We consulted the IR catalogues with the VizieR tool (Ochsenbein, Bauer \& Marcout 2000) at the position of S61 and we extracted the flux densities in the Wide-field Infrared Survey Explorer (WISE) bands W3 and W4 


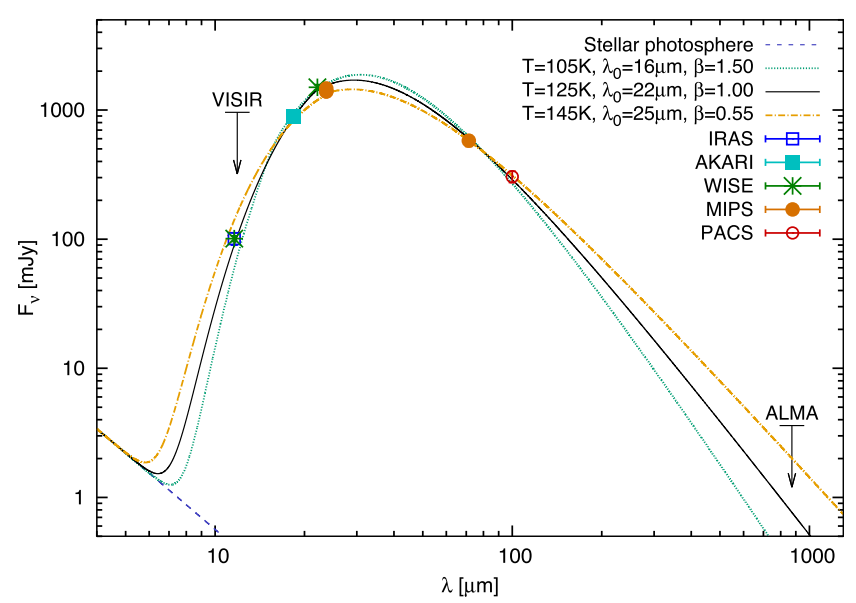

Figure 8. Flux density distribution of S61 derived by using the flux densities in the IR catalogues. The continuum lines represent the fit grey-body functions obtained for three values of the parameter $\beta$. The $3 \sigma$ upper limit spatially integrated flux densities at the VISIR and ALMA observing wavelengths (11.88 and $875 \mu \mathrm{m}$, respectively) are also shown.

(Cutri et al. 2012a,b), AKARI L18W at $18 \mu \mathrm{m}$ (Ishihara et al. 2010a,b), Spitzer MIPS at 24 and $70 \mu \mathrm{m}$ (Ardila et al. 2010a,b), and Herschel PACS at $100 \mu \mathrm{m}$ (Meixner et al. 2013). We fitted a single-temperature grey body with power-law opacity index $\beta$ at longer wavelengths and constant opacity at shorter wavelengths (e.g. Backman \& Paresce 1993),

$F_{v}(\lambda) \propto \tau(\lambda) B_{v}(\lambda), \quad \tau(\lambda)=\left\{\begin{array}{ll}\tau_{0} & \lambda \leq \lambda_{0} \\ \tau_{0}\left(\frac{\lambda}{\lambda_{0}}\right)^{-\beta} & \lambda>\lambda_{0}\end{array}\right.$.

We found a range of characteristic temperatures between $\sim 105$ and $145 \mathrm{~K}$ by varying the parameter $\beta$ (we explored the cases for $\beta=$ $0.55,1$, and 1.5) and $\lambda_{0}$ (between 18 and $25 \mu \mathrm{m}$ ). The modified blackbody that best fits the data is the one represented with a dark continuum line in the figure, with $\beta=1.0$. Note that $\beta=1.0$ implies either the existence of relatively large grains or different dust components (of different temperatures). The latter is usually observed in Galactic LBVNe and the temperature of the dust decreases with increasing distance from the star (e.g. Hutsemekers 1997; Buemi et al. 2017).

According to the flux density distributions in Fig. 8, the expected flux densities $F_{v}$ at the VISIR PAH2_2 and Q1 central wavelengths (11.88 and $17.65 \mu \mathrm{m})$ are: $105 \pm 40$ and $764 \pm 50 \mathrm{mJy}$, respectively. If this emission arises from a point-like source close to the star as observed in other candidate LBVs (e.g. G79.29+0.46; Agliozzo et al. 2014), we would have detected it with VISIR. We deduce then that the dust is spread out over the nebula, at angular scales that our observations were not sensitive to. Similarly, we have derived the expected ALMA flux density at $343 \mathrm{GHz}: 0.8_{-0.5}^{+1.2} \mathrm{mJy}$. Even for the most favourable case for the dust ( $\beta \sim 0.5$, corresponding to optically thick large grains), the sensitivity achieved with only one execution was not sufficient to detect the thermal emission at sub-milllimetre wavelengths. In fact, with an expected flux density of $2 \mathrm{mJy}$ (case $\beta=0.55$ ), spread across 16.7 ALMA synthesized beams, the average brightness would be $0.12 \mathrm{mJy} \mathrm{beam}^{-1}$ and a sensitivity of $40 \mu \mathrm{Jy}_{\text {beam }}{ }^{-1}$ was needed for a $3 \sigma$ detection. Note that the extrapolated flux density at the ALMA frequency is consistent with the upper limit derived from the map: the rmsnoise $(72 \mu \mathrm{Jy})$, integrated over the area corresponding the ionized nebula, yields a $3 \sigma$ upper limit of $\sim 3.6 \mathrm{mJy}$. Using the flux density extracted from the best fit (case $\beta=1.0$ ) at the ALMA frequency $343 \mathrm{GHz}$ (see Fig. 8), we derived a dust mass $M_{\mathrm{d}}=5 \times$ $10^{-3}-2.7 \times 10^{-2} \mathrm{M}_{\odot}$, considering that $M_{\mathrm{d}}=S_{v} D^{2} /\left(B_{v}(T) \kappa_{v}\right)$ and assuming $\kappa_{343 \mathrm{GHz}}=1 \mathrm{~cm}^{2} \mathrm{~g}^{-1}$. This means a low gas-to-dust ratio for the LMC. It suggests that the sub-millimetre emission might be even lower than that computed from the flux density distributions based on the mid- and far-IR data.

The extinction map resembles the dusty nebula around the Galactic LBV IRAS 18576+034 (Buemi et al. 2010). This was also observed with VISIR in the filters PAH2_2 and Q1. They derived for the mid-IR nebula a dust component of temperature ranging from 130 to $160 \mathrm{~K}$. IRAS $18576+034$ has a mid-IR nebula of 7 arcsec diameter, corresponding to $0.35 \mathrm{pc}$ at the distance of $10 \mathrm{kpc}$. It has a physical size that is about half that of S61, but in the sky, the two sources have similar angular size. We rescaled the IRAS 18576+034 VISIR maps to the distance of the LMC and we derived from the maps a mean value of $0.3 \mathrm{mJy} \mathrm{pixel}^{-1}$ in the PAH2_2 filter and 1.3 mJy pixel $^{-1}$ in the Q1 filter. This means that with our VISIR observations, we would have detected at $\sim 3.5 \sigma$ in the PAH2_2 filter image a nebula like IRAS 18576+034. The sensitivity reached in band Q1 would have not been sufficient to detect the nebula. Buemi et al. (2010) derived for IRAS $18576+034$ a dust mass of $\sim 4.5-6.5 \times 10^{-3} \mathrm{M}_{\odot}$ (depending on the assumed dust composition) and Umana et al. (2005) derived a mass of $\sim 2 \mathrm{M}_{\odot}$ for the ionized gas. This suggests that the dust content in the nebula around S61 is similar in mass to that estimated in IRAS $18576+034$. Conversely, the ionized mass in S61 is only a small fraction $(1 / 20$ th) of the mass in the IRAS $18576+034$ nebula, despite S61's nebula diameter $(\sim 1.2 \mathrm{pc})$ being about 3.5 times bigger than IRAS $18576+034(\sim 0.35 \mathrm{pc})$. We recall, however, that IRAS $18576+034$ has an estimated bolometric luminosity higher than $\mathrm{S} 61\left(\log \left(\frac{L}{\mathrm{~L} \odot}\right)=6.4\right.$; Ueta et al. 2001) and the mass, through the mass-loss' quadratic dependence on luminosity, has a stronger effect than the metallicity. The inner shell around Wray 15-751, which has a luminosity similar to S61, extends up to $1 \mathrm{pc}$, similar to S61 and has gas and dust masses of $1.7 \pm 0.6$ and $\sim 3.5 \times 10^{-2} \mathrm{M}_{\odot}$, respectively (Vamvatira-Nakou et al. 2013), more massive than the S61 nebula. This may suggest that S61's mass-loss has been less efficient over time than the mentioned Galactic LBVs. The dust production does not seem significantly different. However, a potential issue for this comparison is the larger uncertainty of the Galactic LBVs distances than those of the Magellanic objects.

\section{CONCLUSIONS}

In this work, we presented high spatial resolution observations from the radio to the mid-IR of the nebula associated with the candidate LBV S61. It was detected only in the centimetre band. The nebula has a morphology resembling a shell as in the optical, but in the radio, there is more sub-structure. The emission mechanism is optically thin free-free, as evidenced by the spectral index map, although there are regions that suggest self-absorption.

We developed and made publicly available a code in PYTHON that permits to model the 3D electron density distribution and to derive the mass in the nebula. We tried different geometries for the shell (truncated Gaussian, constant density, and power-law $n_{\mathrm{e}} \propto r^{-1}$ and $\propto r^{-2}$ ) and we found that at least three of these geometries give similar-quality results. For all the well-fitting models, the derived ionized mass is always about $0.1 \mathrm{M}_{\odot}$, which is an order of magnitude smaller than previous estimates and also a few factors smaller than the mass of similar Galactic objects. The nebula is very likely 
density bounded, meaning that part of the stellar UV flux escapes from the nebula. As an application of our modelled electron density distribution, we also show how to derive the mass-loss history with high temporal resolution ( $\sim 850 \mathrm{yr})$. The derived mass-loss rates are consistent with the empirical mass-loss rate for S61, implying that the nebula was likely formed by stellar winds, rather than eruptive phenomena. The present-day mass-loss is $\lesssim 2 \times 10^{-5} \mathrm{M}_{\odot} \mathrm{yr}^{-1}$.

Based on the extinction map derived from the radio map and the $\mathrm{H}_{\alpha} H S T$ image, we have explored the possibility that the nebular regions with higher spectral index are dusty, by means of highresolution mid-IR and sub-millimetre observations. We did not detect any point-like source, or compact regions associated with the clumps, neither with VISIR nor with ALMA. The fit of the IR flux distribution from space telescope observations suggest the presence of dust with a range of characteristic temperatures of $125 \pm 20 \mathrm{~K}$ and dust mass $M_{\mathrm{d}}$ of $10^{-3}-10^{-2} \mathrm{M}_{\odot}$. Based on the observations with VISIR and ALMA, we exclude that the IR emission arises from a point-like source. The dust producing the IR emission observed by space telescopes must be searched for within the angular scales of the ionized gas $(\sim 1-5$ arcsec $)$. The dust is distributed in an optically thin configuration over the radio nebula, but not uniformly, as shown in the extinction map. The VISIR observations did not reach the required sensitivity to detect such extended thermal emission. With the ALMA observations, we obtained better sensitivity to study thermal emission, but still the nebula was not detected. We estimate that the thermal emission could be detected by deeper ALMA observations in the future, including $7 \mathrm{~m}$ antennas to enhance sensitivity on larger angular scales.

\section{SUPPLEMENTS}

Rhocube (Nikutta \& Agliozzo 2016) is a general-use, stand-alone code and is distributed as such in the following git repository: https://github.com/rnikutta/rhocube. In the spirit of scientific reproducibility, we also share with the reader all scripts and supplementary codes that we have used in this work, specifically the MCMC sampling and Bayesian inference functions that make use of RHocuBe, the functions to compute the ionized mass within a density model and the mass-loss history, and some plotting routines. A git repository for this manuscript, holding all supplementary files including the data FITS files, is accessible at: https://github.com/rnikutta/s61-supplements.

\section{ACKNOWLEDGEMENTS}

The authors wish to thank the referee for their valuable suggestions that helped to improve the presentation of this work. We acknowledge support from FONDECYT grant No. 3150463 (CA) and FONDECYT grant No. 3140436 (RN), and from the Ministry of Economy, Development, and Tourism's Millennium Science Initiative through grant IC120009, awarded to The Millennium Institute of Astrophysics, MAS (CA and GP). We wish to thank the ESO Operations Support Center for the support received for the VISIR data reduction.

This paper makes use of the following ALMA data: ADS/JAO.ALMA\#2013.1.00450.S. ALMA is a partnership of ESO (representing its member states), NSF (USA) and NINS (Japan), together with NRC (Canada) and NSC and ASIAA (Taiwan) and KASI (Republic of Korea), in cooperation with the Republic of Chile. The Joint ALMA Observatory is operated by ESO, AUI/NRAO and NAOJ.
In addition, this research is based on observations made with ESO telescopes at the La Silla Paranal Observatory under programme ID 095.D-0433; makes use of data products from the Wide-field Infrared Survey Explorer, which is a joint project of the University of California, Los Angeles, and the Jet Propulsion Laboratory/California Institute of Technology, funded by the National Aeronautics and Space Administration; made use of the VizieR catalogue access tool, CDS, Strasbourg, France; made use of the NASA/ IPAC Infrared Science Archive (IRSA) that is operated by the Jet Propulsion Laboratory, California Institute of Technology, under contract with the National Aeronautics and Space Administration; made use of the SIMBAD data base, operated at CDS, Strasbourg, France; made use of Montage, funded by the National Aeronautics and Space Administration's Earth Science Technology Office, Computation Technologies Project, under Cooperative Agreement Number NCC5-626 between NASA and the California Institute of Technology. Montage is maintained by IRSA.

\section{REFERENCES}

Abbott D. C., Bieging J. H., Churchwell E., 1981, ApJ, 250, 645

Agliozzo C., Umana G., Trigilio C., Buemi C., Leto P., Ingallinera A., Franzen T., Noriega-Crespo A., 2012, MNRAS, 426, 181 (Paper I)

Agliozzo C., Noriega-Crespo A., Umana G., Flagey N., Buemi C., Ingallinera A., Trigilio C., Leto P., 2014, MNRAS, 440, 1391

Agliozzo C. et al., in press

Ardila D. R. et al., 2010a, ApJS, 191, 301

Ardila D. R. et al., 2010b, VizieR Online Data Catalog, 219

Backman D. E., Paresce F., 1993, in Levy E. H., Lunine J. I., eds, Protostars and Planets III. Univ. Arizona Press, Tucson, p. 1253

Bentley J. L., 1975, Commun. ACM, 18, 9

Bohannan B., Walborn N. R., 1989, PASP, 101, 520

Buemi C. S., Umana G., Trigilio C., Leto P., Hora J. L., 2010, ApJ, 721, 1404

Buemi C. S. et al., 2017, MNRAS, 465, 4147

Clark B. G., 1980, A\&A, 89, 377

Clark J. S., Larionov V. M., Arkharov A., 2005, A\&A, 435, 239

Conti P. S., Frost S. A., 1976, BAAS, 8, 340

Crowther P. A., Smith L. J., 1997, A\&A, 320, 500

Cutri R. M. et al., 2012a, Explanatory Supplement to the WISE All-Sky Data Release Products

Cutri R. M. et al., 2012b, VizieR Online Data Catalog, 2311

Davidson K., Humphreys R. M., 2012, Astrophysics and Space Science Library, Vol. 384, Eta Carinae and the Supernova Impostors. SpringerVerlag, Berlin

Duncan R. A., White S. M., 2002, MNRAS, 330, 63

Fullerton A. W., Massa D. L., Prinja R. K., 2006, ApJ, 637, 1025

Gvaramadze V. V., Kniazev A. Y., Fabrika S., 2010, MNRAS, 405, 1047

Hastings W. K., 1970, Biometrika, 57, 97-109

Higgs L. A., Wendker H. J., Landecker T. L., 1994, A\&A, 291, 295

Humphreys R. M., Davidson K., 1994, PASP, 106, 1025

Humphreys R. M., Weis K., Davidson K., Gordon M. S., 2016, ApJ, 825, 64

Hutsemekers D., 1997, in Nota A., Lamers H., eds, ASP Conf. Ser. Vol. 120, Luminous Blue Variables: Massive Stars in Transition. Astron. Soc. pac., San Francisco, p. 316

Ishihara D. et al., 2010a, A\&A, 514, A1

Ishihara D. et al., 2010b, VizieR On-line Data Catalog, 2297

Jiménez-Esteban F. M., Rizzo J. R., Palau A., 2010, ApJ, 713, 429

Kraemer K. E. et al., 2010, AJ, 139, 2319

Lamers H. J. G. L. M., Snow T. P., Lindholm D. M., 1995, ApJ, 455, 269

Lang C. C., Johnson K. E., Goss W. M., Rodríguez L. F., 2005, AJ, 130, 2185

McMullin J. P., Waters B., Schiebel D., Young W., Golap K., 2007, in Shaw R. A., Hill F., Bell D. J., eds, ASP Conf. Ser. Vol 376, Astronomical Data 
Analysis Software and Systems XVI. Astron. Soc. Pac., San Francisco, p. 127

Meixner M. et al., 2013, AJ, 146, 62

Metropolis N. et al., 1953, J. Chem. Phys., 21, 6

Nazé Y., Rauw G., Hutsemékers D., 2012, A\&A, 538, A47

Nikutta R., 2012, PhD thesis, Univ. Kentucky. Available at: http://uknowledge.uky.edu/physastron_etds/8/

Nikutta R., Agliozzo C., 2016, rnikutta/rhocube: 1.0.1. Available at: https://doi.org/10.5281/zenodo.160465

Ochsenbein F., Bauer P., Marcout J., 2000, A\&AS, 143, 23

Oskinova L. M., Hamann W.-R., Feldmeier A., 2007, A\&A, 476, 1331

Panagia N., Felli M., 1975, A\&A, 39, 1

Paron S., Combi J. A., Petriella A., Giacani E., 2012, A\&A, 543, A23

Pasquali A., Langer N., Schmutz W., Leitherer C., Nota A., Hubeny I., Moffat A. F. J., 1997, ApJ, 478, 340

Pasquali A., Nota A., Clampin M., 1999, A\&A, 343, 536

Pauldrach A. W. A., Puls J., 1990, A\&A, 237, 409

Pottasch S. R., 1984, Astrophys. Space Sci. Libr., 107, 335

Sault R. J., Teuben P. J., Wright M. C. H., 1995, in Shaw R. A., Payne H. E., Hayes J.J.E., ASP Conf. Ser. Vol. 77, Astronomical Data Analysis Software and Systems IV. Astron. Soc. Pac., San Francisco, p. 433

Schaller G., Schaerer D., Meynet G., Maeder A., 1992, A\&AS, 96, 269

Smith N., Owocki S. P., 2006, ApJ, 645, L45

Smith L. J., Nota A., Pasquali A., Leitherer C., Clampin M., Crowther P. A., 1998, ApJ, 503, 278

Stahl O., 1986, A\&A, 164, 321

Trotta R., 2008, Contemp. Phys., 49, 71

Ueta T., Meixner M., Dayal A., Deutsch L. K., Fazio G. G., Hora J. L., Hoffmann W. F., 2001, ApJ, 548, 1020

Umana G., Buemi C. S., Trigilio C., Leto P., 2005, A\&A, 437, L1

Umana G., Buemi C. S., Trigilio C., Leto P., Hora J. L., 2010, ApJ, 718, 1036

Umana G., Buemi C. S., Trigilio C., Leto P., Hora J. L., Fazio G., 2011a, Bull. Soc. R. Sci. Liege, 80, 335

Umana G., Buemi C. S., Trigilio C., Leto P., Agliozzo C., Ingallinera A., Noriega-Crespo A., Hora J. L., 2011b, ApJ, 739, L11

Umana G. et al., 2012, MNRAS, 427, 2975

Vamvatira-Nakou C., Hutsemékers D., Royer P., Nazé Y., Magain P., Exter K., Waelkens C., Groenewegen M. A. T., 2013, A\&A, 557, A20

Vamvatira-Nakou C., Hutsemékers D., Royer P., Cox N. L. J., Nazé Y., Rauw G., Waelkens C., Groenewegen M. A. T., 2015, A\&A, 578, A108 van Genderen A. M., 2001, A\&A, 366, 508

Vink J. S., de Koter A., 2002, A\&A, 393, 543

Vink J. S., Gräfener G., 2012, ApJ, 751, L34

Vink J. S., de Koter A., Lamers H. J. G. L. M., 1999, A\&A, 350, 181

Vink J. S., de Koter A., Lamers H. J. G. L. M., 2000, A\&A, 362, 295

Wachter S., Mauerhan J., van Dyk S., Hoard D. W., Morris P., 2011, Bull. Soc. R. Sci. Liege, 80, 291

Walborn N. R., 1977, ApJ, 215, 53

Walborn N. R., 1982, ApJ, 256, 452

Weis K., 2003, A\&A, 408, 205

Weis K., 2008, in Hamann W.-R., Feldmeier A., Oskinova L. M., eds, Clumping in hot-star Winds. Universitatsverlag Potsdam, Potsdam, p. 183

Zacharias N., McCallon H. L., Kopan E., Cutri R. M., 2005, in Engvold O., ed., Highlights of Astronomy, Vol. 13. Astron. Soc. Pac., San Francisco, p. 603

\section{APPENDIX A: BRIEF INTRODUCTION TO RHOCUBE}

\section{A1 Principles}

RHocube computes a 3D density field $\varrho(x, y, z)$ on a Cartesian, right-handed grid, with $x$ pointing to the right, $y$ pointing up, and $z$ pointing to the viewer. The grid resolution is set by the user upon model instantiation. Several models with density distributions of common interest are provided, and new ones can be easily added by the user. At the time of writing, the (mnemonically named) provided models are: PowerLawShell, TruncatedNormalShell, ConstantDensityTorus, ConstantDensityDualCone, Helix3D. The latter takes as the envelope parameter the imaginary surface on which the helical tube spirals (dual cone or cylinder). The models are implemented as PYTHON classes and all inherit basic functionality, such as e.g. 3D rotations and $x, y$ offsets, from a common class Cube.

Every model has a number of free parameters, e.g. for PowerLawShell, the inner and outer radii $r$ in and rout and the radial powerlaw index pow. Two lateral offsets xoff and yoff to de-centre the density distribution in the image plane are available to all the models, as are the rotation angles tiltx, tilty, tiltz, which, when provided, rotate the entire 3D density field about the respective axes. They are, of course, ineffectual for spherically symmetric density distributions. Fig. A1 shows a few examples of integrated maps that can be computed with the code.

\section{A2 Usage}

The workflow with RHOcuBE is straightforward:

(i) Instantiate a model (e.g. PowerLawShel1);

(ii) Call the instance with a set of parameter values;

(iii) Retrieve/access the 3D density cube and/or 2D $z$-integrated image.

Instantiating a model generates the Cartesian grid (with requested resolution) and provides it with general methods to manipulate the density distribution (e.g. 3D rotations and shifts in $(x, y, z))$. Once the model is created, it can be called any number of times with a set of numerical arguments that are the values that the free model parameters should assume. Each call computes the corresponding 3D density field and also integrates that field along the $z$-axis, storing the resulting $2 \mathrm{D}$ image as a member of the model instance. If the smoothing parameter was set to a float value, then $\varrho(x, y, z)$ will be smoothed with a 3D Gaussian kernel (see Section A3.2). Listing A2 shows a simple example instantiating and calling a simple model.

Listing 1: instantiating and calling a model.

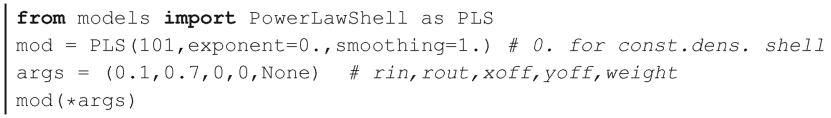

The resulting $\varrho(x, y, z)$ is now in mod.rho (as 3D array) and the summation along $z$ direction is in mod. image. If you wanted to vary the parameters of this model:

$\operatorname{args}=(0.3,0.5,0,0.3$, None) $\#$ rin, rout, xoff, yoff, weight
$\bmod ($ *args $)$

If a transform function is provided during instantiation, it will be applied to $\varrho(x, y, z)$ before integration (see Section A3.1). If during calling, the weight parameter is set to a float value, the sum $\sum_{i} \varrho(x, y, z)$ (of the possibly transformed $\varrho(x, y, z)$ ) over all voxels will be normalized to that value. Listing 2 shows an example.

Listing 2: Using transform function and weight parameter.

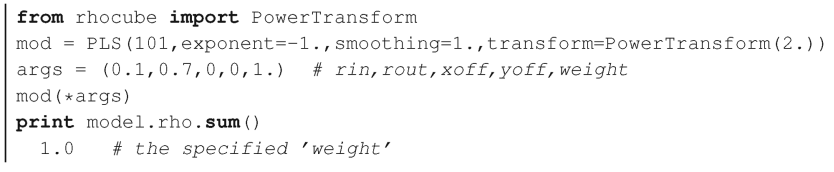



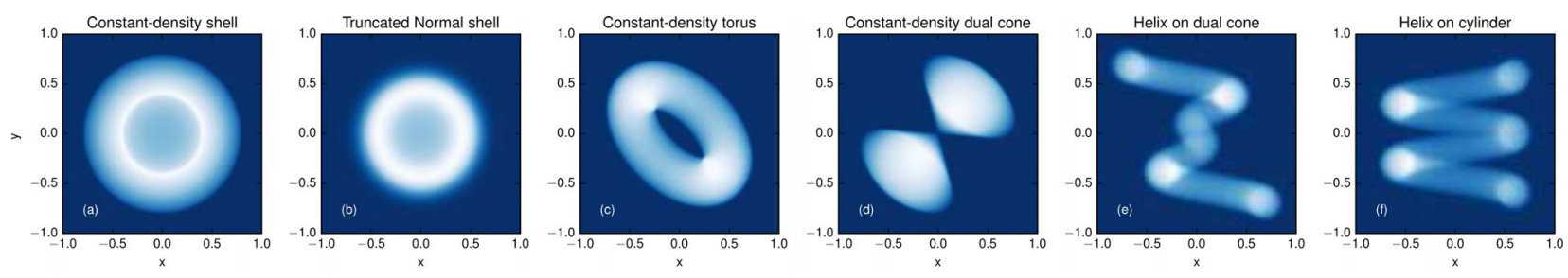

Figure A1. Example gallery of some $z$-integrated model density maps that can be generated with RHocuBE. $x$ and $y$ are in relative linear units. The colour map is scaled to the image maxima. (a) Constant-density shell with four free parameters: inner and outer shell radii and the centre offsets xoff and yoff. (b) A truncated Gaussian-density shell with six free parameters: radius, width of the Gaussian, left and right cut-off radii, two offsets. (c) A constant-density torus with six free parameters: radius, cross-section, two tilt angles, two offsets. (d) A dual-cone with six free parameters: height, opening angle, two tilts, two offsets. (e) Helical tube on the surface of a dual cone, with the free parameters' radius at top of the cone, number of turns taken from origin, tube diameter, three tilts, two offsets. (f) Like (e) but on the surface of a cylinder. An arbitrary combination of models, with relative total masses as free parameters, is also possible.

\section{A3 Special methods}

\section{A3.1 The 'transform' function}

An optional transform function $f(\varrho)$ can be passed as an argument when creating a model instance. The transforms are implemented as simple PYTHON classes. A transform will be applied to the density field before $z$-integration, i.e. $\int \mathrm{d} z f(\varrho(x, y, z))$ will be computed. In our case, for instance, squaring of the electron number density before integration is required by equation (1). If the supplied $f(\varrho)$ class also provides an inverse function, e.g. $f^{-1}=\sqrt{\cdot}$, when $f=(\cdot)^{2}$, then the entire $3 \mathrm{D}$ cube $\varrho(x, y, z)$ with correct scaling can also be computed and accessed by the user. Some common transform classes are provided with RHOCUBE, e.g. PowerTrans form, which we use for the squaring mentioned above (with argument pow $=2$ ) or LogTransform that computes a base-base logarithm of $\varrho(x, y, z)$. Another provided transform is GenericTrans form that can take any parameter-free NUMPY function and inverse (the defaults are func $=$ ' $\sin ^{\prime}$ and inversefunc $=$ ' arcsin' $)$. Custom transform functions can be easily added.

\section{A3.2 Smoothing of the 3D density field}

Upon instantiating a model, the smoothing parameter can be specified. If smoothing is a float value, it is the width (in standard deviations) of a 3D Gaussian kernel that $\varrho(x, y, z)$ will be convolved with, resulting in a smoothed 3D density distribution. Smoothing does preserve the total $\sum_{i} \varrho(x, y, z)$, where $i$ runs over all voxels. smoothing $=1.0$ is the default and does not alter the resulting structure significantly. If smoothing=None, no smoothing will be applied.

\section{A4 Providing own density distributions}

The Cube class provides two convenience objects and methods to compute the 3D density $\varrho(x, y, z)$, which can (but do not need to) be utilized by the actual model upon instantiation. The two methods are computeR and buildkdtree.

\section{A4.1 X,Y,Z coordinate arrays}

By default, 3D Cartesian coordinate grids X,Y,and Z are computed upon instantiation of the Cube class and each holds the $x$ or $y$ or $z$ coordinates of the voxel centres, in fractional units of a cube with extent $[-1,1]$ along every axis. They can be used to compute arbitrary dependences $\varrho(x, y, z)$.

\section{A4.2 Distance array}

If compute $\mathrm{R}=\mathrm{True}$ is passed to $\mathrm{Cube}$ during model instantiation, then the class will also compute a $3 \mathrm{D}$ radius grid $\mathrm{R}(\mathrm{x}, \mathrm{Y}, \mathrm{z})$, i.e. a cube of npix ${ }^{3}$ voxels, each holding its own radial distance from the cube centre. This $R$ cube can then be used inside the model to compute a distance-dependent density as $\varrho(R)$. This method is used in all azimuthally symmetric models that come with RHOCUBE, e.g. PowerLawShell and TruncatedNormalshell.

Below, we show in a simple example how one can construct a custom 3D density model that computes a spherically symmetric $\varrho(R)$ that varies as the cosine of distance, i.e. $\varrho(R) \propto \cos (R)$.

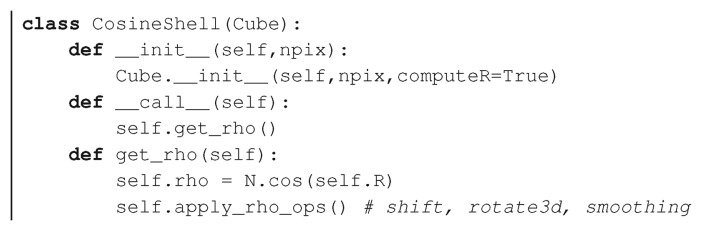

You can then use it simply like this:

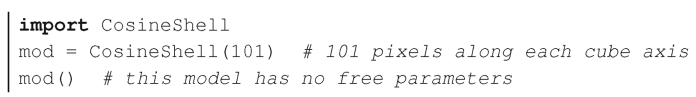

Please see the built-in model classes for more details and ideas.

\section{A4.3 k-d tree}

RHOCUBE also supports non-symmetric or irregular density distributions. One example might be the (also provided) model for a helix that winds along some prescribed parametric curve. For fast computation of all voxels within some orthogonal distance from the parametric curve (i.e. within a 'tube'), we utilize the second helper method in Cube, namely a k-d tree (Bentley 1975). The Helix3D model works like this:

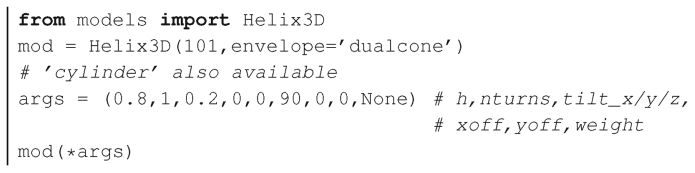

Note that the initial building of the k-d tree is a $\mathcal{O}\left(n \log ^{2} n\right)$ operation. The subsequent lookups are then much faster. Please see the Helix3D class for more details of the implementation. 


\section{APPENDIX B: BAYESIAN PARAMETER INFERENCE}

\section{B1 Conditional probability and Bayes' theorem}

Estimating the most likely physical parameters (inputs) of a model whose output is compared to observed data is by far the most common scenario of Bayesian statistics. In our case, the inputs are the geometrical parameters of the modelled 3D electron density distribution $n_{\mathrm{e}}(x, y, z)$ and the outputs are the modelled EM maps. They are compared to the observed EM maps of a given LBV shell.

We must vary the free model parameters, with the objective of minimizing the deviation of the resulting model EM map and the data EM map (for instance, $\chi^{2}$, if the data errors can be assumed Gaussian). We desire not only to estimate the best-fit parameters, but to quantify their uncertainty or the confidence that we can have in the results. The most natural approach to this common parameter estimation problem is Bayesian inference. Using notation borrowed from statistical literature, Bayes' theorem

Posterior $\equiv p(\vec{\theta} \mid \boldsymbol{D})=\frac{p(\vec{\theta}) p(\boldsymbol{D} \mid \vec{\theta})}{p(\boldsymbol{D})} \equiv \frac{\text { Prior } \times \text { Likelihood }}{\text { Evidence }}$

provides a straightforward prescription how to compute the joint posterior probability distribution (PDF) $p(\vec{\theta} \mid \boldsymbol{D})$ of a possibly multivariate vector of model parameters $\vec{\theta}=\left(\theta_{1}, \theta_{2}, \ldots\right)$, given the observed data vector $\boldsymbol{D}$. The posterior PDF distribution is simply a product of a prior PDF $p(\vec{\theta})$ (i.e. any knowledge we may have of the model parameter distribution before introducing the data) with the likelihood that the given parameter values generate a model that is compatible with the data. For normally distributed errors, the likelihood is $p(\boldsymbol{D} \mid \vec{\theta}) \propto \exp \left(-\chi^{2} / 2\right)$ (see e.g. Trotta 2008).

The evidence $p(\boldsymbol{D})$ in equation (B1) is the normalization (integral of the multidimensional posterior PDF), ensuring that the total probability be unity. For the sole purpose of parameter inference, it is not necessary to compute the evidence explicitly, since it does not change the shape of the posterior PDF. It is instead sufficient to re-normalize the posterior to unit volume a posteriori. Thus, for parameter inference, only the relation $p(\vec{\theta} \mid \boldsymbol{D}) \propto p(\vec{\theta}) p(\boldsymbol{D} \mid \vec{\theta})$ is relevant. Of particular interest for the interpretation of results are the marginalized 1D posterior distributions, each integrated over all model parameters but the one in question

$p\left(\theta_{i} \mid \boldsymbol{D}\right)=\int \mathrm{d} \theta_{1} \mathrm{~d} \theta_{1} \ldots \mathrm{d} \theta_{i-1} \mathrm{~d} \theta_{i+1} \ldots \mathrm{d} \theta_{N} p(\boldsymbol{D} \mid \vec{\theta})$.
Every $\theta_{i} \in \vec{\theta}$ is one of the free model parameters and every $\theta_{j} \neq \theta_{i}$ is a so-called nuisance parameter when computing the marginalized posterior PDF of $\theta_{i}$. Hence, the common expression 'marginalize over the nuisance parameters'. In our application, the marginalized posteriors are shown in panels (2)-(5) in Fig. 5. Panel (6) shows the posterior PDF of a derived quantity, which, in the Bayesian approach, is trivial to compute and in the 'classical' approach, impossible.

\section{B2 MCMC sampling}

The $N$ model parameters span an $N$-dimensional volume that grows exponentially with the number of parameters. It very quickly becomes impractical to sample the entire volume. Fortunately, for many problemsm only small sub-volumes are relevant, i.e. the likelihood is only high in small regions of the parameter volume. Several methods to sample preferentially these highly significant regions have been proposed. The best known is probably MCMC sampling. A particularly straightforward MCMC formalism is the Metropolis-Hastings algorithm introduced by Metropolis et al. (1953) and later generalized by Hastings (1970). It can be shown that the proposal joint PDF from which the algorithm samples eventually converges towards the sought-after target posterior PDF. MCMC thus generates a chain of samples (for every model parameter $\left.\theta_{i}\right)$, whose histogram is the marginalized posterior $p\left(\theta_{i} \mid \boldsymbol{D}\right)$.

A common way to characterize the marginal PDFs is to compute the median ( 0.5 percentile of the cumulative distribution function, $\mathrm{CDF}$ ) and as the confidence interval (or 'credible interval') the boundaries of an inter-percentile range. For Gaussian PDFs, this can be the $\pm 1 \sigma$ interval around the median, i.e. the range $[0.158-$ 0.841] of the CDF. For (slightly) asymmetric PDFs, an inter-quartile range is often used, i.e. [0.25-0.75]. While the posteriors in our application are not always Gaussian, for consistency, we will report as the confidence interval the $\pm 1 \sigma$ range around the median throughout.

This paper has been typeset from a $\mathrm{T}_{\mathrm{E}} \mathrm{X} / \mathrm{L} \mathrm{T} \mathrm{E} \mathrm{X}$ file prepared by the author. 